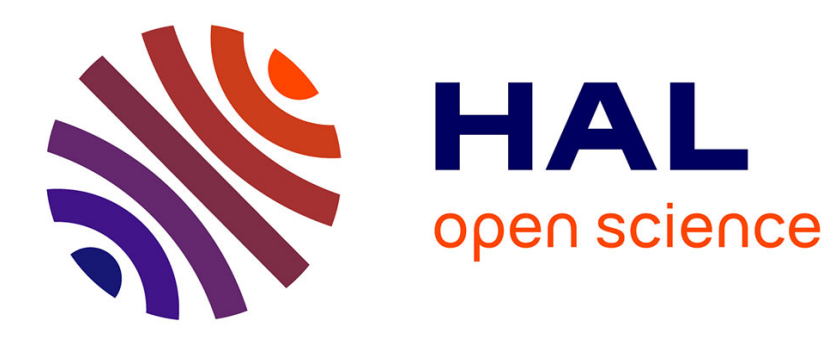

\title{
Revision of fossil Bibionidae (Insecta: Diptera) from French Oligocene deposits
}

\author{
John Skartveit, André Nel
}

\section{To cite this version:}

John Skartveit, André Nel. Revision of fossil Bibionidae (Insecta: Diptera) from French Oligocene deposits. Zootaxa, 2017, 4225 (1), pp.1-83. 10.11646/zootaxa.4225.1.1 . hal-01471927

\section{HAL Id: hal-01471927 \\ https://hal.sorbonne-universite.fr/hal-01471927}

Submitted on 20 Feb 2017

HAL is a multi-disciplinary open access archive for the deposit and dissemination of scientific research documents, whether they are published or not. The documents may come from teaching and research institutions in France or abroad, or from public or private research centers.
L'archive ouverte pluridisciplinaire HAL, est destinée au dépôt et à la diffusion de documents scientifiques de niveau recherche, publiés ou non, émanant des établissements d'enseignement et de recherche français ou étrangers, des laboratoires publics ou privés. 


\title{
Revision of fossil Bibionidae (Insecta: Diptera) from French Oligocene deposits
}

\author{
JOHN SKARTVEIT \& ANDRÉ NEL
}

John Skartveit, NLA University College Bergen, P.O. Box 74 Sandviken, N-5812 Bergen, Norway, email: John.Skartveit@NLA.no

André Nel, Institut de Systématique, Évolution, Biodiversité, ISYEB - UMR 7205 - CNRS, MNHN, UPMC, EPHE, Muséum national d'Histoire naturelle, Sorbonne Universités, 57 rue Cuvier, CP 50, Entomologie, F-75005, Paris, France,e-mail: anel@mnhn.fr

\begin{abstract}
All available material of fossil Bibionidae from French Oligocene localities, including the German locality of Kleinkembs right next to the border with France, is revised, and the species redescribed. Several publications (notably Heer 1856, Oustalet 1870 and Théobald 1937) have dealt with this material but there is a large degree of duplication leading to numerous synonyms. In addition, many of the named species have been assigned to the wrong genus. We have found seven species of Penthetria, eleven species of Plecia, seven species of Bibio and a single species of Dilophus in the material of previously named species. Three new species, Penthetria luberonica sp.n., Bibio aquaesextiae sp.n. and Bibiodes provincialis sp.n. are described from French Oligocene localities. The following new combinations are proposed: Penthetria claripennis (Théobald, 1937), Penthetria gigantea (Théobald, 1937), Penthetria graciliventris (Théobald, 1937), Penthetria longiventris (Théobald, 1937), Penthetria nervisinuata (Théobald, 1937), Penthetria subterranea (Théobald, 1937), Plecia morio (Heer, 1849), Bibio major (Oustalet, 1870), Dilophus luteipennis (Théobald, 1937). Many of the species occur from several outcrops, and distinctive faunas can be recognized from Early Oligocene (e.g., Célas and Monteils), Middle Oligocene (e.g., Céreste and Bois d'Asson) and Late Oligocene (e.g. Aix-en-Provence and the German outcrop of Rott). The late Oligocene localities also share some species with the Early Miocene locality of Radoboj, Croatia, but none with the younger locality of Öhningen, Southern Germany. For a number of named species, the type material is poorly preserved and cannot be recognized at the species level, these are commented on and assigned to the lowest taxonomical level to which they can be identified with certainty. The type materials of a number of species appear to be lost; these are placed to genera to the extent that this is possible from the original descriptions and illustrations. Bibio nigripennis Théobald, 1937, nec Brunetti, 1913 is a primary junior homonym and the name must be replaced. The species is moved to the genus Plecia and renamed Plecia theobaldi nom.n.
\end{abstract}

Key words: Penthetria, Plecia, Bibio, Bibiodes, Dilophus, France, new species, new combination

\section{Introduction}

Bibionid flies are very abundant in some European lacustrine deposits from the Oligocene (Collomb et al. 2008), in particular the tropical and subtropical bibionid genus Plecia Wiedemann, 1828 is very abundant in several deposits. A large number of nominal species have been described from these deposits. Evenhuis (1994) listed 74 species of Plecia, in addition 28 species of Penthetria, and 57 species of Bibio from European Oligocene fossils. However, a large number of these species 
descriptions are based on quite incomplete specimens, or the characters used to define species are of taphonomical and not phylogenetic significance. For instance, the relative size and shape of the abdomen is commonly used as a character (e.g. Théobald 1937 p. 331). Flies found in lacustrine deposits have fallen into water. As the fly partly decomposes, the abdomen will swell and get bloated, while the more solidly sclerotised parts (head, thorax and legs) will maintain their shape. Taphonomic compression has also an important effect on the shape of the abdomen, more or less compressed depending on the pressure that the dead animal had to support when embedded in or between the laminae. Thus the apparent size and shape of the abdomen will depend on the time it took before the dead fly was covered by sediment, not on any trait of the living specimen. This was pointed out by Statz (1943) who nevertheless used the relative length of the abdomen as an important character. There is some evidence that a few fossil bibionids were actually brachypterous (Skartveit \& Pika 2014), these can however be recognized on their deviant wing shape and venation. Théobald (1937) also used the shape of the abdomen together with the colour of the insect body and wings as diagnostic characters. A further difficulty is the fact that in the same outcrop (or even lamina), the preservation of the colours can vary greatly in relation to the epigenisation of the insect.

In modern bibionids descriptions of new species are normally based on male specimens, and characters of the male terminalia are considered very important. In contrast, the majority of species descriptions in fossil bibionids were based on females, partly because these were more numerous in the deposits and partly because they, being somewhat more robust than the males, tended to be better preserved. Distinguishing modern species based on females alone is very difficult in areas where numerous species occur, this is much more so with fossil specimens. Statz (1943) suggested that all descriptions of new fossil bibionid species should be based on females since they are more abundant in impression fossil materials, and the majority of previously described species were obviously based on female specimens. While this would seem a reasonable compromise given the state of the material and the published species, we choose not to follow it since that would differ radically from the strategy chosen in the morphologically similar, recent species, and since it would preclude the use of the systematically very useful male terminalia. Bibionids being often quite abundant in Tertiary fossil insect faunas, there will often be well-preserved males available for study, although they tend to be outnumbered by the females (Skartveit \& Pika 2014). A problem with interpreting male terminalia in fossils is that important components, particularly the gonostylus, have three-dimensional shapes and may appear rather different when viewed from different angles (See Collomb et al. 2008 for illustrative examples). Modern specimens and amber fossils may be rotated for comparable views, but this is not possible with compression fossils which are basically rather two-dimensional. Thus the terminalia of male bibionids, though well preserved, may be difficult to interpret. One apparently useful character is the length of the gonostylus compared to the width of the hypopygium. This should be used with great caution, though, since the apparent width of the hypopygium is highly dependent on the degree of flattening. It is probably better to base comparisons on the absolute length of the gonostylus. In the subfamily Bibioninae good species-level distinguishing characters are often found in the antennae, legs and wings (pile colour may also be useful but is hardly ever preserved in compression fossils), so distinguishing species based on females is usually achievable. However, the marked sexual dimorphism causes problems in associating male and female specimens. In some cases, it is possible to find laminae with a quantity of males and females obviously of the same species; they died during a swarm. This phenomenon is rather frequent in the Oligocene of Céreste (France). In Bibioninae, species differences in the male terminalia, though constant, are often so slight that they can rarely be used in fossil specimens and only exceptionally well-preserved specimens show these characters with sufficient clarity. 
European Oligocene bibionids were described by Heer (1849, 1856), Heyden \& Heyden (1865), Oustalet (1870, 1872), Brongniart (1876, 1878), Meunier (1915a, b), Théobald (1937), Statz (1943) and Gentilini (1993). Apparently, none of the above authors thoroughly studied the type material of previous publications before proceeding to describe new species, although Théobald (1937) included numerous species described by Heer, the Heydens and Oustalet in his work. This, together with reliance on apparent characters which originate from taphonomy and not the insect's morphology, has led to a proliferation of species names in this group. The present paper is an attempt to revise the Oligocene bibionids described from French localities. We have examined all type material which we could locate, and also several thousand non-type specimens from localities in France. Also included are specimens from the German locality of Kleinkembs, which is less than a kilometer from the border with France and was included in the Théobald's (1937) monograph on French Oligocene insects. It is likely that a number of species are shared between the localities in France and the Oligocene localities in Germany, in particular, Rott and Enspel (Heyden \& Heyden 1865, Statz 1943, Wedmann 1998), but these are not considered in the present paper except for the species described by Heyden \& Heyden (1865), the types of which we have examined.

\section{Material and methods}

\section{Collections}

The bulk of the material studied comes from the Museum National d'Histoire Naturelle, Paris (MNHN), which has a collection of about 5000 bibionid fossils from French Oligocene deposits. We have also studied type and non-type specimens from numerous other collections as listed below. Numerous type specimens, particularly for species described by Théobald (1937), are not in any of these collections. We have not included nominal species for which we have not found type material (either labeled as types, recognisable from label data given in the original descriptions or recognisable by illustrations in the original descriptions), except for the species described by Gentilini (1993), for which the descriptions are sufficient for an unambiguous identification of the species, for Penthetria graciliventris (Heer) which has a highly characteristic habitus, and for Plecia morio (Heer) which stands out among the species recorded from Aix-en-Provence due to its large size.

\section{Collections included}

EEFN École des Eaux et Forêts de Nancy, France; the material is currently stored in MNHN

EM École Nationale Superieure des Mines de Paris, France; the material is stored in the University Claude Bernard Lyon 1, Lyon, France.

ETHZ Swiss Federal Institute of Technology, Zurich, Switzerland

FSL University Claude Bernard Lyon 1, Lyon, France

MHNM Museum d'Histoire Naturelle de Marseille, Marseille, France

MNHN Museum National d'Histoire Naturelle, Paris, France

MPP Musee de Paléontologie de Provence, Marseille, France

NHMUK Natural History Museum, London, U.K.

NHMW Naturhistorisches Museum, Vienna, Austria 
NMB Naturhistorisches Museum, Basel, Switzerland

RGL Réserve Géologique du Lubéron, Apt, France (coll. Nel); the material is temporarily stored in MNHN

SLJG Steiermärkisches Landesmuseum Joanneum, Graz, Austria

SMNK Staatliches Museum für Naturkunde, Karlsruhe, Germany

UPMC Université Pierre et Marie Curie, Paris, France

\section{Fossil deposits}

France has a large number of fossil sites including several Oligocene sites containing insect fossils. The localities in France were discussed by Collomb et al. (2008, p. 162-163). All deposits are lacustrine, most of them marl and limestone deposits. Some show traces of marine fauna, though not generally together with the bibionid fossils.

\section{Céreste}

The most abundant material originates from Céreste, Vaucluse $\left(43.9^{\circ} \mathrm{N}, 5.6^{\circ} \mathrm{E}\right)$. The locality is from the Middle Oligocene (33.9-28.4 MYR BP). Specimens from Céreste have probably been deposited onto a sticky biofilm, and are mostly complete and reasonably well preserved. The material from Céreste include more than 2600 fossil bibionid specimens, the vast majority of which are currently in Coll. Nel, MNHN, to be deposited in RGL.

\section{Vitrolles}

There is a material of approximately 90 specimens in FSL from Vitrolles-en-Lubéron, Vaucluse $\left(43.8^{\circ}\right.$ $\mathrm{N}, 5.6^{\circ} \mathrm{E}$ ) with the nearby site of La Tuiliére. The specimens are well preserved. No species have been described previously from this locality.

\section{Aix-en-Provence}

Several outcrops at Aix-en-Provence, Bouches-du-Rhône $\left(43.5^{\circ} \mathrm{N}, 5.5^{\circ} \mathrm{E}\right)$ contain abundant insect fossils. These sites, which are Late Oligocene (28.4-23.0 MYR) age, were among the first insect fossil sites discovered, and were discussed already by Lyell \& Murchison (1829). The material from Aix is rather variable in appearance, few specimens show much remains of organic material but some are nice relief impressions. We have examined $>1600$ fossil bibionid specimens from Aix, the vast majority of which are stored in MNHN. There are, however, numerous types from Aix-en-Provence in BMNH, ETHZ and FSL. There is also a substantial material from Aix in the EM collection, stored at FSL.

\section{Bois d'Asson}

The localities at Bois d'Asson and Dauphín, Alpes-de-Haute-Provence $\left(43.9^{\circ} \mathrm{N}, 5.8^{\circ} \mathrm{E}\right)$ are Middle Oligocene (33.9 - 28.4 MYR) in age. The layers with insects are the same in the two outcrops, which are $2 \mathrm{~km}$ away from each other. There are 188 fossil bibionid specimens from these localities in 
MNHN, to be transferred to RGL. Though the site is geographically very close to Céreste and of approximately the same age, the species assemblage seems to be to some extent different from that found at Céreste. Species from Bois d'Asson were described by Gentilini (1993).

\section{Chadrat}

The locality of Chadrat with the nearby sites St.Saturnin and St. Marguerite, Puy-de-Dôme $\left(45.7^{\circ} \mathrm{N}\right.$, $3.1^{\circ} \mathrm{E}$ ) is Middle Oligocene (33.9-28.4 MYR) in age. There are 109 specimens from this locality in MNHN. Most are females and relatively poorly preserved, but this fauna appears to be very similar to Céreste. Théobald (1937) figured a single Bibio specimen from Chadrat but no species appear to have been described from this site.

\section{Camoins}

The locality of Camoins-les-Bains, Bouches-du-Rhône $\left(43.3^{\circ} \mathrm{N}, 5.5^{\circ} \mathrm{E}\right)$ with the nearby site of Aubagne is from the Late Oligocene (28.4-23.0 MYR). There are 33 specimens from this site in MNHN and at least seven specimens in MPP. Théobald (1937) described one fossil bibionid species from Camoins, the type of this appears unfortunately to be lost.

\section{Monteils}

The locality of Monteils with the nearby site St. Hippolyte de Caton, Gard $\left(44.1^{\circ} \mathrm{N}, 4.2^{\circ} \mathrm{E}\right)$ is of Late Eocene (c. 35 MYR). There are 173 specimens from this site in MNHN, most of them well preserved. The fauna is distinctively different from the younger sites. One bibionid species was described from Monteils by Théobald (1937).

\section{Célas}

The locality of Célas, Gard $\left(44.1^{\circ} \mathrm{N}, 4.2^{\circ} \mathrm{E}\right)$ is of Late Eocene to Early Oligocene age (37.2-33.9 MYR). The fauna appears to be very similar to the nearby and similar in age site at Monteils. Numerous species were described from the site by Théobald (1937). Material from Célas is sparse in the collections we have examined. We found four specimens in MNHN, six in MHNM and a single specimen in FSL. In addition there is a single plate with over 100, mostly poorly preserved, specimens of Bibio celasensis in MHNM.

\section{Barbeiras}

The locality of Barbeiras, Vaucluse, is of Early Oligocene (33.9-28.4 MYR) age. There are 50 specimens from this locality in MNHN, mostly poorly preserved females. The fauna appears to differ little from that of Céreste. No species have been described from this locality.

\section{Corent}

The locality of Corent, Puy-de-Dôme $\left(45.7^{\circ} \mathrm{N}, 3.2^{\circ} \mathrm{E}\right)$ is from the Middle Oligocene. Numerous species were described from the site by Oustalet (1870), unfortunately some of the material appears to be lost. There are 20 specimens from Corent in MNHN.

\section{Ronzon}

The locality of Ronzon, Haute-Loire $\left(45.0^{\circ} \mathrm{N}, 3.9^{\circ} \mathrm{E}\right)$ is from the Early Oligocene (33.9-28.4 MYR BP). One single paratype of Plecia splendida Théobald, 1937 originates from this site. 


\section{Kleinkembs}

Although the locality is actually in Germany, the locality of Kleinkembs, Baden $\left(47.7^{\circ} \mathrm{N}, 7.5^{\circ} \mathrm{E}\right)$ is very close to the French border and it is natural to include it in this review, as did Théobald (1937) in his review of French Oligocene insects. The site is of Middle Oligocene (33.9-28.4 MYR) age. There are 180 bibionid specimens from Kleinkembs in NMB, many of them well preserved. Numerous species were described from the site by Théobald (1937).

\section{Study of the specimens}

Specimens were photographed using a digital camera (Olympus E3) equipped with a macro lens (Olympus Zuiko Digital $35 \mathrm{~mm}$. f 2.8) for later study. Total length or wing length (whichever was more accessible) were measured and other measurements were later collected from the photos using these as standards. Thorax length was measured from the anterior margin of pronotum to posterior edge of mediotergite, thorax width as the distance between wing bases in dorsal view. Abdominal width was measured across the third tergite. Length of curved structures (wing veins, gonostyli) was measured along the curve. Wing width was measured at the widest point, which is generally at midwing for Penthetria and Plecia spp., a little more basad for Bibioninae. As usual with fossil insects, the amount of information that can be extracted from a specimen is highly variable, some type specimens are near perfect and others are barely (or not at all) recognizable. For this reason the amount of information available in the description will vary from species to species, it is not possible to be fully consistent except by rejecting a large fraction of the previously described species. We have measured the size of various structures where that is possible, but the measurements given in the descriptions of the various species are frequently collected from different specimens. For this reason, it is not possible to calculate ratios between different measurements from the data as here presented. Drawings were prepared based on the digital photos, and, in some cases, from camera lucida sketches made using a stereo microscope.

\section{Wing vein terminology}

Wing vein terminology follows the traditional terminology of Merz \& Haenni (2000). An alternative classification (Wootton \& Ennos 1989) has revised the terminology of the seventh vein reaching the wing edge $\left(\mathrm{CuA}_{1}\right.$ according to Merz \& Haenni), classifying this as a branch of $\mathrm{M}\left(\mathrm{as} \mathrm{M}_{4}\right)$, we have chosen not to follow this since the vein in Bibionidae is connected to $\mathrm{M}$ only through a fine, transverse vein, whereas a strong longitudinal vein connects it with $\mathrm{CuA}$. The most posterior, long vein is interpreted as CuP in accordance with Wootton \& Ennos (1989). Vein $\mathrm{A}_{1}$ is rarely possible to see in compression fossils and accordingly we do not include it in the descriptions. The humeral crossvein, Subcosta and the crossvein $\mathrm{M}-\mathrm{Cu}$ are also frequently faint and difficult to see. They are included in the descriptions where we have been able to trace them, but if they are not mentioned in a description it does not mean that they are absent unless it is specifically stated so.

\section{Results}

\section{Key to the fossil Bibionidae from French Oligocene deposits}

Unless otherwise stated, the key is applicable for both sexes.

1. Radial sector branched, legs slender

Penthetriinae and Pleciinae 
Radial sector undivided, legs more or less thickened Bibioninae

2. Vein $\mathrm{R}_{2+3}$ long, horizontal to oblique

Vein $\mathrm{R}_{2+3}$ shorter, oblique to vertical

Plecia

$-9$

3. Larger species, body length mostly $12-13 \mathrm{~mm}$, female wing light brown, posterior veins colourless. Male gonostylus slender.

Penthetria gigantea

Smaller species, body length mostly $<12 \mathrm{~mm}$

4. Vein $\mathrm{R}_{2+3}$ bent near base, sinuous, very long.

Penthetria nervisinuata

Vein $\mathrm{R}_{2+3}$ straight or evenly curved, shorter.

5. Body long and slender (Fig. 10), thorax convex

Penthetria graciliventris

Body not conspicuously long and slender

6. Wing dark brown fumose.

Penthetria longiventris

Wing hyaline to light brown fumose.

7. Male gonostylus pointed apically. Female sternite VIII without appendages.

$-8$

Male gonostylus blunt apically. Females unknown.

Penthetria subterranea

Female sternite VIII with a pair of digitiform appendages. Males unknown

\section{Penthetria lugens}

8. Wing with basal part of radial sector steep, forming a $45^{\circ}$ angle with $\mathrm{R}_{1}$

Penthetria claripennis

Wing with basal part of radial sector forming a less than $30^{\circ}$ angle with $\mathrm{R}_{1}$

\section{Penthetria luberonica}

9. Wing dark brown fumose, body dark.

Wing light brown fumose to hyaline, body usually lighter coloured

10. Larger species, body length $14-15 \mathrm{~mm}$. Female head conspicuously small.

\section{Plecia morio}

Smaller species, body length 7-10.5 mm. Female head not conspicuously small. - 11

11. Wing veins black throughout, pterostigma black and distinctive. $\mathrm{R}_{2+3}$ straight, may have a kink in middle part.

Plecia lygaeoides 
Wing veins not black throughout, pterostigma indistinctive. $\mathrm{R}_{2+3}$ curved.

\section{Plecia bucklandi}

12. Male gonostylus hooked and sharply pointed, epandrium with three finger-like projections. Female unknown. Plecia larguensis

Male gonostylus not hooked and pointed, or male unknown $-13$

13. Slender species, legs and abdomen pale brown.

Rbust species, legs and abdomen dark brown.

14. Wing narrow, hyaline, R-veins brown, very distinctive. Larger species, wing length $9 \mathrm{~mm}$. Flagellum 9-segmented.

Plecia rhenana

Wing wider, R-veins not darker than more posterior veins. Mostly smaller species. Flagellum often with fewer than 9 segments.

15. Larger species, male wing length at least $5.8 \mathrm{~mm}$. Male gonostylus not sharply pointed, apically thickened nor with a rounded basal lobe.

Plecia livida

Smaller species, male wing length less than $5.8 \mathrm{~mm}$. Male gonostylus sharply pointed, apically thickened or with a rounded basal lobe.

$-16$

16. Male gonostylus apically pointed.

Plecia assonensis

Male gonostylus apically rounded.

17. Male gonostylus slender, basally with a rounded lobe projecting dorsally.

Plecia maimensis

Male gonostylus clavate, apically thickened, without basal lobe.

Plecia conica

18. Larger species, body length $8.5-10.5 \mathrm{~mm}$, female sternite VIII with hook-shaped appendage.

Plecia inflata

Smaller species, body length less than $8 \mathrm{~mm}$, female sternite VIII without hook-shaped appendage

- 19

19. Wing with pterostigma conspicuous, $\mathrm{R}_{2+3}$ distinctly curved.

Plecia lapidaria

Wing with pterostigma inconspicuous, $\mathrm{R}_{2+3}$ nearly straight.

Plecia foersteri

20. Fore tibia cylindrical with mesal spines, crossvein R-M longer than basal Radial sector. Smaller species, body length 5.5-6 mm.

Dilophus luteipennis

Fore tibia spindle-shaped with apical spine, R-M shorter than basal Radial sector. Mostly larger species.

21. Crossvein R-M nearly to entirely obliterated, basal Radial sector reaches vein M. Male head conspicuously small.

Crossvein R-M distinctive. Male head normal-sized. 
22. Veins Rs and M merged for some distance. Smaller species, 5.5-7.5 mm. Antenna stout with 5 -segmented flagellum.

Bibiodes provincialis

Veins Rs and M meet but do not merge. Larger species, 8-12 mm. Antenna slender with 10segmented flagellum.

Bibio major

23. Wing veins blackish, pigmented throughout, pterostigma blackish.

Posterior wing veins colourless, pterostigma less distinctive.

24. Vein $\mathrm{CuA}_{1}$ apically straight.

Bibio spectabilis

Vein $\mathrm{CuA}_{1}$ apically hooked.

Bibio curtisii

25. Veins R-M and basal radial sector more or less equal in length.

Crossvein R-M short, basal radial sector two to three times as long as it. - 27

26. Larger (female 10-13 mm), slender species. Male hind tibia strongly clavate.

Bibio aquaesextiae

Smaller (female 6.7-9 mm), robust species. Male unknown.

Bibio dislocatus

27. Wing yellowish fumose with concolourous veins and pterostigma. Larger species, $8-13 \mathrm{~mm}$.

Bibio baumbergeri

Wing light brown with dark brown R-veins and dark pterostigma. On average smaller species, 6-9 mm.

Bibio celasensis

\section{Genus Penthetria Meigen, 1803}

While it is straightforward to distinguish the genera Penthetria and Plecia in recent species, this is not always so with fossils. In the recent species, it is most convenient to identify the genera based on the shape of the vein $\mathrm{R}_{2+3}$, which is long and horizontal in Penthetria, short and steep in Plecia. However, in fossil species the shape of $R_{2+3}$ (sometimes named $R_{3+4}$ ) varies continuously (Rice 1959) and it is not possible to draw a non-arbitrary line between Penthetria and Plecia wing venation. Handlirsch (1908) chose to put all fossil bibionids with forked Radial sector in the genus Penthetria, this may be warranted given the limited information available on the morphology of most of the fossil species. However, some fossil species are quite typical of the genus Plecia. In recent species, male terminalia in Plecia spp., though highly variable, is almost always decidedly different from the plesiomorphic pattern invariably found in Penthetria species. However, many fossil species that habitually conform to Plecia have plesiomorphic terminalia, with digitiform gonostyli which are attached distally on the gonapophysis and are directed mesally (e.g., Skartveit 2009). Typical Penthetria males can still be recognized on the very robust, pilose, curved gonostyli, but this is apparent only in very well preserved fossils, whereas a large proportion of the named species have been described from females or poorly-preserved males. One often has to resort to general habitus to assign these species to genus since characters of the terminalia are not available. Penthetria species are generally larger (most species more than $10 \mathrm{~mm}$ ), more gracile with markedly longer legs, slender male abdomens, the wings are longer, slender in the males and notably large in the female, usually fumose. Plecia species are 
generally smaller (most species smaller than $10 \mathrm{~mm}$ ), more robust with shorter legs, male abdomen stockier, and shorter wings which are often hyaline.

\section{Penthetria claripennis (Théobald, 1937) comb.n. (Figs. 1-5, 72-77)}

Plecia claripennis Théobald, 1937: 228. (Basel)

Plecia variegata Théobald, 1937: 234. Syn.n.

Plecia varia Théobald, 1937: 234. Syn.n.

Plecia difficilis Théobald, 1937: 234-235. Syn.n.

Plecia graciosa Théobald, 1937: 235. Syn.n.

Material examined: Holotype (female) of Plecia claripennis NMB F1382, (fig. 1). Holotype (male) of Plecia variegata NMB F1405, (fig. 2). Paratype (male) of Plecia variegata NMB F1406. Holotype (male) of Plecia varia NMB F1407, (fig. 3). Paratypes (males) of Plecia varia NMB F455, F1417/1, 1418. Holotype (male) of Plecia difficilis NMB F1408, (fig. 4). Paratypes (6 males) of Plecia difficilis NMB F1420/1, F1421/1-5. Holotype (male) of Plecia graciosa NMB F1409, (fig. 5) (part and counterpart), paratypes (3 males) of Plecia graciosa NMB F1423, F1424, F1425/1, F1425/2. Additional material (10 males): NHB F1416/2-6, F1419/1-4, (2 females) NMB F1415/1, F1416/1.

Localities: Kleinkembs.

Diagnosis: A smallish Penthetria (7-9.5 mm). Male wing light brownish fumose, vein $\mathrm{R}_{2+3}$ slightly curved, basal part of Rs forming approximately $45^{\circ}$ angles with $\mathrm{R}_{1}$ and $\mathrm{M}$. Male epandrium trapezoidal, gonostylus basally robust, distally slender and sharp. Female wing hyaline.

\section{Redescription:}

Male (fig. 77): Total length 7.7-9.5 mm (N=28).

Head: Length 0.9-1.5 mm ( $\mathrm{N}=23)$, width $0.9-1.2 \mathrm{~mm}(\mathrm{~N}=6)$. Ocellar tubercle tall and prominent. Complex eye without conspicuous pile. Antenna relatively short, flagellum 8-segmented? Palp rather long and slender.

Thorax: Length 1.8-2.7 mm ( $\mathrm{N}=28)$, width 1.7-2.0 mm ( $\mathrm{N}=5)$. Black, convex, rather short and wide, sulci prominent. Haltere light brown.

Wing (fig. 72): Length 6.3-8.8 mm ( $\mathrm{N}=22)$, width 2.0-2.9 $\mathrm{mm}(\mathrm{N}=11)$. Yellowish brown fumose, veins light brown, pterostigma indistinct. Costa extends slightly beyond apex of $\mathrm{R}_{4+5}$. Humeral vein present, fine and oblique. Subcosta long and distinctive. $\mathrm{R}_{2+3}$ straight with a slight bend in middle. Pterostigma light brownish, ill-defined. Basal section of Rs steep, forming a $45^{\circ}$ angle with $R_{1}$.

Crossvein R-M oblique. Fork of M relatively short, not much longer than stem beyond R-M crossvein. Wing vein measurements in Table 1.

Legs: Brown, rather long and slender, femorae slightly clavate. Fore femur 2.1-2.7 mm ( $\mathrm{N}=8)$, fore tibia 2.1-3.1 $\mathrm{mm}(\mathrm{N}=7)$, hind femur 2.6-3.4 $\mathrm{mm}(\mathrm{N}=18)$, hind tibia 2.2-3.4 $\mathrm{mm}(\mathrm{N}=14)$. 
Abdomen: Length 4.9-7.2 mm ( $\mathrm{N}=27)$, width 0.8-1.6 mm ( $\mathrm{N}=11)$, yellowish-brown, cylindrical, moderately long. Pile not conspicuous.

Terminalia (figs. 73-75): Width of hypopygium 0.83-1.03 mm (N=14). Epandrium trapezoidal, mesally slightly incurved. Gonostylus length $0.32-0.55 \mathrm{~mm}(\mathrm{~N}=14)$, curved, basally robust, apically sharply pointed.

Female: Total length $7.0-11.0 \mathrm{~mm}(\mathrm{~N}=3)$.

Head length 0.9-1.2 $\mathrm{mm}(\mathrm{N}=2)$, not well preserved. Ocellar tubercle prominent.

Thorax: Length 2.0-2.5 mm ( $\mathrm{N}=3)$, width $1.6 \mathrm{~mm}(\mathrm{~N}=1)$. Black, convex.

Wing (fig. 76): Length $8.0-8.8 \mathrm{~mm}(\mathrm{~N}=3)$, width $2.9 \mathrm{~mm}(\mathrm{~N}=1)$, almost devoid of pigment in specimens at hand. R-veins distinctive, venation approximately as in male. Wing vein measurements in Table 1 .

Legs: Brown, relatively long and slender. Femorae a little clavate.

Abdomen: Length 4.5-7.0 mm ( $\mathrm{N}=2)$, width 1.8-2.3 mm ( $=2)$, dark brown, cylindrical, rather short and stocky.

Terminalia: Cercus rather slender and pointed.

Remark: The abdomen appears to be shrunk in one of the female specimens, bloated in another, which explains the apparently large variation in total and abdomen lengths.

\section{Penthetria gigantea (Théobald, 1937) comb.n. (Figs. 6-9, 78-80)}

Plecia gigantea Théobald, 1937: 226-227.

Plecia crassiventris Théobald, 1937: 227. Syn.n.

Plecia miegi Théobald, 1937: 232-233. Syn.n.

Plecia luteipennis Théobald, 1937: 233. Syn.n.

Material examined: Holotype (female) of Plecia gigantea NMB F1380, (fig. 6). Paratypes (females) of Plecia gigantea NMB F460, F1388. Holotype (female) of Plecia crassiventris NMB F1381, (fig. 7). Paratypes (females) of Plecia crassiventris NMB F1389/1, F1389/2. Holotype (male) of Plecia miegi NMB F1399 (fig. 8), part + counterpart. Paratypes (males) of Plecia miegi NMB F1400, F1401. Holotype (male) of Plecia luteipennis NMB F1402/F2420 (fig. 9) (part and counterpart of same specimen). Paratype (male) of Plecia luteipennis NMB F1413.

Localities: Kleinkembs.

Diagnosis: A large $(8-13.5 \mathrm{~mm})$ and slender Penthetria with relatively long and slender legs. Antennal flagellum probably 8-segmented. Male epandrium subtriangular, gonostylus conical, apically slender. Wing yellowish fumose in male, brownish in female. Vein $\mathrm{R}_{2+3}$ nearly straight with a small bend in middle. 


\section{Redescription:}

Male: Total length $8.0-13.5 \mathrm{~mm}(\mathrm{~N}=4)$. Entirely black.

Head: Length 1.1-1.5 mm ( $\mathrm{N}=4)$, width $1.1 \mathrm{~mm}(\mathrm{~N}=2)$. Antennal flagellum cylindrical, approximately 8 -segmented.

Thorax: Length 2.1-3.0 mm ( $\mathrm{N}=4)$, width 1.7-2.4 mm ( $\mathrm{N}=2)$. Haltere dark brown.

Wing (fig. 78): Length 9.1-11.6 mm ( $\mathrm{N}=3)$, width 3.2-3.4 mm ( $=2)$, length/width $=3.02-3.40$ $(\mathrm{N}=3)$. Light brown fumose with brown $\mathrm{R}$-veins, posterior veins colourless. Costa extends some distance beyond apex of $\mathrm{R}_{4+5}$. Humeral vein present, Subcosta long and horizontal. $\mathrm{R}_{2+3}$ rather straight, oblique. Pterostigma inconspicuous. Basal part of Rs subparallel to M. Fork of M relatively long. Wing vein measurements in Table 1.

Legs: relatively long and slender, dark brown. Femorae slightly clavate. Femur 1 length $3.2-3.6 \mathrm{~mm}$ $(\mathrm{N}=2)$, tibia1 length 3.3-3.6 mm $(\mathrm{N}=2)$, femur 3 length 3.4-4.5 $\mathrm{mm}(\mathrm{N}=3)$, tibia 3 length $3.8-4.3 \mathrm{~mm}$ $(\mathrm{N}=2)$.

Abdomen: Length 4.6-9.6 mm (N=5), width 1.0-1.4 mm (N=2). Brown, slender, cylindrical. Abdominal pile rather short.

Terminalia (fig. 80): Epandrium rather small, subtriangular. Gonostylus robust, almost straight, sharply pointed. Hypopygium width $1.3-1.5 \mathrm{~mm}(\mathrm{~N}=2)$, gonostylus length $0.64-0.67 \mathrm{~mm}(\mathrm{~N}=2)$.

Female: Total length $9.2-13.5 \mathrm{~mm}(\mathrm{~N}=4)$. Entirely black.

Head: Length $0.9-1.2 \mathrm{~mm}(\mathrm{~N}=3)$, width $0.7-1.0 \mathrm{~mm}(\mathrm{~N}=2)$. Relatively small, oval, longer than broad. Complex eyes do not protrude. Antennal flagellum cylindrical, probably eight-segmented.

Thorax: Length 2.8-4.6 mm (N=6), width 2.3-3.2 mm (N=4). Black. Sulci deep and conspicuous. Pile not conspicuous. Haltere yellowish-brown.

Wing (fig. 79): Length 9.4-11.5 mm (N=6), width 3.0-3.8 mm ( $=3)$, length/width = 2.95-3.77 $(\mathrm{N}=4)$. Brown fumose. Radial veins light brown, strong, more posterior veins fine and inconspicuous. Pterostigma invisible. Venation as in male. Wing vein measurements in Table 1.

Legs: Dark brown. Long and slender, femorae slightly clavate. Tibia 3 length $5.1 \mathrm{~mm}(\mathrm{~N}=1)$.

Abdomen: Length 5.9-8.1 mm ( $\mathrm{N}=3)$, width 2.5-3.2 mm (N=4). Dark brown, cylindrical. Pile not conspicuous.

Terminalia: Cercus two-segmented as usual for genus, rather small, pointed.

\section{Penthetria graciliventris (Théobald, 1937) comb.n. (Figs. 10, 81-84)}

Plecia graciliventris Théobald 1937: 138.

The type of this species was said to be in Marseille, but we could not find it and it is possibly lost. However, some specimens from Monteils of a very distinctive, slender habitus fit perfectly Théobald (1937)'s drawing (Pl. XI, Fig. 5) and description.

Material: 3 males (MNHN Monteils 35, Fig. 10, Monteils 55, Monteils 57.), 1 female: MNHN Monteils 25. 
Localities: Célas, Monteils.

Diagnosis: A medium-sized species $(8-9.5 \mathrm{~mm})$ with an unusually slender habitus. The species is easily recognized by its very gracile body - the superficial habitus of a female of this species is more like a male of a typical bibionid, with a long, slender abdomen. The specimens at hand are of a uniform, brown colour. The strongly convex thorax gives this species a hunch-back appearance. Male gonostylus rather small, curved and pointed, epandrium small, subquadrate with a mesal indentation.

\section{Redescription:}

Male. Total length 8.3-9.0 mm ( $\mathrm{N}=3)$. Dark brown, likely black in life.

Head (fig. 84): Length 1.0-1.1 mm (N=3). Brown, rounded, complex eye rather small and rounded. Antenna rather short, flagellum possibly 7 -segmented. Ocellar tubercle small.

Thorax: Length 2.0-2.1 mm (N=3). Short and strongly convex. Haltere light brown.

Wing (fig. 81): Length 6.1-6.9 mm (N=3), width 1.8-2.2 mm ( $=3)$, length/width $=2.78-3.32(\mathrm{~N}=3)$. Rather long and slender, light brown fumose, anterior area a little darker. Costa extends to nearly halfway between apices of $\mathrm{R}_{4+5}$ and $\mathrm{M}_{1}$. Humeral vein present, Subcosta relatively short, terminating midway between crossvein $\mathrm{R}-\mathrm{M}$ and origin of $\mathrm{R}_{2+3}$. R-veins brown, posterior veins colourless. Pterostigma invisible. Vein $\mathrm{R}_{2+3}$ slightly sinuous, $\mathrm{R}_{4+5}$ distally distinctively curved. Basal part of Rs subparallel to M. Wing vein measurements in Table 1 .

Legs: Brown, slender. Fore femur $1.8 \mathrm{~mm}(\mathrm{~N}=1)$, fore tibia $1.9 \mathrm{~mm}(\mathrm{~N}=1)$, hind femur 2.3-2.8 mm $(\mathrm{N}=3)$, hind tibia $2.4-2.7 \mathrm{~mm}(\mathrm{~N}=2)$.

Abdomen: Length 6.6-6.8 mm (N=3). Long and narrow, cylindrical.

Terminalia (fig. 83): Epandrium quite small, suboval. Gonostyli relatively small, digitiform, curved and pointed.

Female: Total length $9.5 \mathrm{~mm}(\mathrm{~N}=1)$.

Head: Width $1.3 \mathrm{~mm}$.

Thorax: Length $2.9 \mathrm{~mm}$. Shape apparently as in male. Haltere pale brownish.

Wing (fig. 82): Length $7.8 \mathrm{~mm}$, width $2.8 \mathrm{~mm}$, length/width $=2.63$. Light brown fumose, veins brown, pterostigma indistinctive. Costa extends to half-way between apices of $\mathrm{R}_{4+5}$ and $\mathrm{M}_{1}$. Humeral vein present, Subcosta relatively long. $\mathrm{R}_{2+3}$ nearly straight. Posterior veins fine, not possible to trace in specimen at hand.

Legs: Slender but not particularly long, femorae slightly clavate. Tibial spurs needle-thin. Fore femur $2.3 \mathrm{~mm}$, fore tibia $2.1 \mathrm{~mm}$, hind femur $2.5 \mathrm{~mm}$, hind tibia $3.0 \mathrm{~mm}$.

Abdomen: Length $7.2 \mathrm{~mm}$, width $2.1 \mathrm{~mm}$. Long and cylindrical, not apically tapered.

Terminalia: Abdominal tip rounded. 


\section{Penthetria longiventris (Théobald, 1937) comb.n. (Figs. 11, 85-89)}

Plecia longiventris Théobald, 1937: 133-134.

Plecia superba Théobald, 1937: 134-135, nec Meunier, 1915. Syn.n.

Material examined: Paratype female FSL 391901 (fig. 11). This is the specimen depicted by Théobald (1937: XI: 8), from Célas.

Additional material: MNHN Monteils 11 (female), Monteils 13 (female), Monteils 16 (male), Monteils 20 (female), Monteils 21 (female), Monteils 26 (male), Monteils 50 (female), Monteils 51 (male), Monteils 108 (female), Monteils 145 (female), Monteils 161 (female).

\section{Localities: Célas, Monteils.}

Diagnosis: A medium-sized (8.5-11.5 mm) Penthetria. Most specimens are of a uniform, brown colour. Antennal flagellum 9-segmented. Male gonostylus very robust, short, sharply pointed. Female head rounded, ocellar tubercle not very prominent, complex eye relatively small.

\section{Redescription:}

Male: Total length $8.5-10.5 \mathrm{~mm}(\mathrm{~N}=3)$. Entirely dark brown, probably black in life.

Head (fig. 86): Length $1.4 \mathrm{~mm}(\mathrm{~N}=1)$, width $1.6 \mathrm{~mm}(\mathrm{~N}=1)$. Complex eye without conspicuous pile. Antenna relatively long, segments not possible to count in any of the specimens at hand.

Thorax: Length $2.1 \mathrm{~mm}(\mathrm{~N}=2)$. Haltere pale.

Wing: Length $6.8 \mathrm{~mm}(\mathrm{~N}=1)$. Hyaline to light brown fumose, veins concolourous with membrane, pterostigma invisible. Costa extends a little beyond apex of $\mathrm{R}_{4+5}$. Humeral vein present, Subcosta fine and long. Vein $\mathrm{R}_{2+3}$ straight, oblique. $\mathrm{R}_{4+5}$ distally curved. Fork of $\mathrm{M}$ long and rounded.

Legs: Entirely slender. Fore femur $2.8 \mathrm{~mm}(\mathrm{~N}=1)$, fore tibia $2.7 \mathrm{~mm}(\mathrm{~N}=1)$. Hind femur $2.5 \mathrm{~mm}$ $(\mathrm{N}=1)$, hind tibia $2.6 \mathrm{~mm}(\mathrm{~N}=1)$.

Abdomen: Length 5.7-6.9 mm (N=3), width 1.1-1.3 mm (N=2). Slender, slightly conical.

Terminalia (fig. 87): Hypopygium width 0.9-1.1 mm ( $\mathrm{N}=2)$, gonostylus length $0.38-0.45 \mathrm{~mm}(\mathrm{~N}=2)$. Gonostylus very robust, sharply pointed.

Female: Total length 8.5-11.5 mm (N=5). Dark brown.

Head (figs. 88-89): Length 1.13-1.39 mm ( $=3$ ), width 0.99-1.34 mm ( $=4)$. Rounded triangular, widest near hind corners. Complex eye not prominent. Antenna relatively elongate, flagellum 9segmented, $0.77 \mathrm{~mm}$ long $(\mathrm{N}=1), 0.15 \mathrm{~mm}$ wide $(\mathrm{N}=1)$.

Thorax: Length 2.4-3.2 mm (N=2), width 1.9-2.6 mm ( $\mathrm{N}=2)$. Haltere brown.

Wing (fig. 85): Length 7.1-11.5 mm ( $=7)$, width $3.2 \mathrm{~mm}(\mathrm{~N}=1)$, length/width $=2.70(\mathrm{~N}=1)$, brown fumose, anterior part slightly darkened, veins dark brown, coloured throughout wing. Costa extends a 
little beyond apex of $\mathbf{R}_{4+5}$. Pterostigma not conspicuous. $\mathbf{R}_{2+3}$ almost straight, slightly curved. $\mathbf{R}_{4+5}$ distally curved. Fork of $\mathrm{M}$ long and rounded. $\mathrm{CuA}_{2}$ curved basad in distal part. Vein measurements in Table 1.

Legs: Dark brown, relatively short, slender. Fore femur 2.1-3.2 $\mathrm{mm}(\mathrm{N}=2)$, fore tibia 2.4-3.0 mm $(\mathrm{N}=2)$, hind femur 2.4-3.5 $\mathrm{mm}(\mathrm{N}=3)$, hind tibia 2.3-4.0 $\mathrm{mm}(\mathrm{N}=4)$.

Abdomen: Brown. Length 5.3-8.3 mm (N=5), width 2.2-3.2 mm (N=5).

Terminalia: Tergite IX mesally with a deep, U-shaped excavation. Cerci rather small, two-segmented, slender.

\section{Penthetria luberonica sp.n. (Figs. 12-13, 90-94)}

Material examined: Holotype (male) FSL 140048b Paratype (males) FSL 140050, FSL 140053g.

Additional material (7 males 20 females): FSL 140009, 140010, 140021, 140023, 140027, 140034, 140036, 140038, 140041, 140046, 140048a, 140049, 140053, 140279, 140307, 140312.

Etymology: The species is named after the Lubéron Massive, where the outcrop is situated.

Localities: Vitrolles.

Diagnosis: A small Penthetria, total length 7-9 millimetres. Antennal flagellum 9-segmented. Wing light brownish fumose, $\mathrm{R}_{2+3}$ of medium length, slightly sinuate. Male gonostylus robust, curved and pointed, epandrium dome-shaped. Most similar to $P$. claripennis, but recognized by basal part of Rs being nearly parallel to $\mathrm{M}$.

\section{Description:}

Male: Total length 7.0-9.5 mm (N=8).

Head (fig. 91): Length 0.92-1.21 mm ( $\mathrm{N}=5)$, width 0.96-1.21 mm ( $\mathrm{N}=5)$. Brown. Complex eye rather small, subspherical, prominent. Flagellum 9-segmented, length $0.47-0.65 \mathrm{~mm}(\mathrm{~N}=3)$.

Thorax: Length 2.0-2.7 mm ( $=8)$, width $1.8 \mathrm{~mm}(\mathrm{~N}=1)$. Dark brown, presumably black in life. Haltere pale brown.

Wing (fig. 90): Length 5.3-7.0 mm ( $\mathrm{N}=7)$, width 2.0-2.8 mm ( $\mathrm{N}=7)$, length/width =2.52-2.82 ( $\mathrm{N}=7)$. Light brown fumose, anterior part slightly darker, pterostigma inconspicuous. Veins light brown throughout. Costa extends to half-way between apices of $\mathrm{R}_{4+5}$ and $\mathrm{M}_{1}$. Humeral vein present. Subcosta relatively strong, terminating in Costa just above origin of $R_{2+3}$. Basal part of Rs subparallel to $M$, crossvein R-M nearly vertical. $\mathrm{R}_{2+3}$ of medium length, nearly straight to slightly sinuous. Fork of $\mathrm{M}$ rounded. Fork of $\mathrm{CuA}$ relatively short, just a little longer than stem. Wing vein measurements in Table 1. 
Legs: Pale brown, not well preserved in any of the specimens at hand.

Abdomen: Length 4.4-6.4 mm ( $\mathrm{N}=7)$, width 0.9-1.0 mm ( $\mathrm{N}=4)$. Brown, quite slender.

Terminalia (fig. 92): Hypopygium width $0.58-0.93 \mathrm{~mm}(\mathrm{~N}=6)$. Gonostylus length $0.43-0.47 \mathrm{~mm}$ $(\mathrm{N}=2)$. Blackish-brown. Epandrium presumably dome-shaped. Gonostylus robust, apically curved and sharply pointed.

Female: Total length 6.7-8.2 mm (N=11).

Head (fig. 94): Length $0.67-0.76 \mathrm{~mm}(\mathrm{~N}=2)$, width $0.67-0.94 \mathrm{~mm}(\mathrm{~N}=8)$. Dark brown, presumably black in life. Flagellum probably 9-segmented, length 0.54-0.62 mm (N=3).

Thorax: Length 1.9-2.4 mm ( $\mathrm{N}=12)$, width 1.5-1.9 mm ( $\mathrm{N}=7)$. Dark brown, presumably black in life. Haltere pale brownish.

Wing (fig. 93): Length 6.1-7.5 mm ( $\mathrm{N}=12)$, width 2.6-3.0 $\mathrm{mm}(\mathrm{N}=11)$, length/width $=2.29-2.57$ $(\mathrm{N}=11)$. Light brownish, slightly darkened anteriorly, pterostigma indistinctive. Humeral vein present, subcosta distinctive, long and straight. Costa extends to nearly half-way between apices of $\mathrm{R}_{4+5}$ and $\mathrm{M}_{1}$. Basal part of Radial sector subparallel to $\mathrm{M} . \mathrm{R}_{2+3}$ oblique, nearly straight. Fork of $\mathrm{M}$ rounded. Wing vein measurements in Table 1. Fork of $\mathrm{CuA}$ not much longer than stem. CuP distinctive, straight. Wing vein measurements in Table 1.

Legs: Not well preserved in any of the specimens at hand. Pale brown, tarsi a little darker. Fore tibia length $1.8-1.9 \mathrm{~mm}(\mathrm{~N}=4)$.

Abdomen: Length 4.7-6.1 mm ( $\mathrm{N}=11)$, width 1.5-2.3 mm ( $=8)$. Brown, apically darkened.

Terminalia: Dark brown, presumably black in life. Tergite VIII rectangular with slightly protruding hind corners. Sternite VIII mesally with a small, rounded indentation.

\section{Penthetria lugens (Oustalet, 1870) (Figs. 14-15, 95-97)}

Protomyia lugens Oustalet, 1870: 142-143.

Plecia cf. lugens (Oustalet, 1870); Théobald, 1937: 227.

Penthetria lugens (Oustalet, 1870); Handlirsch, 1908: 959.

Material examined: Holotype (female) MNHN R 06666.

Additional material: 2 females (MNHN B 24412, B 24518). 5 females (NHB F2208, F2230, F2323, F2410, F2413). 1 female (FSL 391923).

\section{Localities: Corent, Céreste, Kleinkembs}

The holotype specimen (fig. 12) is in very poor condition, wing venation and most details are impossible to see. Collomb et al. (2008) classified the type as Insecta, incerta sedis, though it is recognizable as a Plecia or Penthetria female. However, the diagnostic appendages on gonapophysis VIII are visible, confirming that the holotype is conspecific with Penthetria lugens sensu Théobald, 1937. 
Diagnosis: A medium-sized, slender Penthetria. Body brownish, wings brown fumose. $\mathrm{R}_{2+3}$ slightly sinuous and oblique. On ventral side of abdomen gonapophysis VIII has a pair of digitiform appendages.

\title{
Redescription:
}

Male unknown

Female (fig. 13) Total length $8.3-11.5 \mathrm{~mm}(\mathrm{~N}=4)$.

Head (fig. 96): Length 0.93-1.11 mm (N=3), width 1.01-1.27 mm (N=3). Dark brown, probably black in life. Antennal flagellum probably 5- or 6-segmented.

Thorax: Length 2.0-2.6 mm ( $=4)$, width $2.0 \mathrm{~mm}(\mathrm{~N}=2)$. Dark brown, probably black in life. Haltere yellowish-brown.

Wing (fig. 95): Length 8.6-9.4 mm ( $=4)$, width 2.9-3.3 mm ( $=4)$, length/width $=2.87-3.34(\mathrm{~N}=4)$. Brown fumose, R-veins brown, posterior veins concolourous with membrane. Costa extends nearly to apex of $\mathrm{M}_{1}$. Humeral vein present, Subcosta fine and short, terminating well before crossvein R-M. Pterostigma indistinctive. $\mathrm{R}_{2+3}$ with a bend in basal third, otherwise nearly straight, oblique. $\mathrm{R}_{4+5}$ apically nearly straight. Posterior veins not possible to see in specimens at hand. Wing vein measurements in Table 1.

Legs: Brown, slender. Fore femur $2.0 \mathrm{~mm}(\mathrm{~N}=1)$, fore tibia $2.2 \mathrm{~mm}(\mathrm{~N}=1)$, hind femur $2.6 \mathrm{~mm}(\mathrm{~N}=1)$, hind tibia $2.7 \mathrm{~mm}(\mathrm{~N}=1)$.

Abdomen: Length 6.1-7.6 mm ( $\mathrm{N}=4)$, width 1.7-2.9 mm ( $\mathrm{N}=3)$. Light brown, relatively long and narrow, spindle-shaped.

Terminalia (fig. 97): Gonapophysis VIII with a digitiform appendage on each side. Cerci twosegmented, relatively long.

\section{Penthetria nervisinuata (Théobald, 1937) comb.n. (Figs. 16-17, 98-102)}

\author{
Plecia nervisinuata Théobald 1937: 407 \\ Bibiopsis funebris Heer in Heer \& Gaudin, 1861: 153. (nec Penthetria funebris Meigen, 1804) \\ (nomen nudum) \\ A medium-sized Penthetria, rather stocky for the genus, entirely black as usual for the genus. The \\ shape of vein $R_{2+3}$ is characteristic: it is vertical at base, then turns $90^{\circ}$ and runs parallel to $R_{4+5}$ in the \\ basal half, then turns forwards, meeting Costa at an almost $45^{\circ}$ angle.
}

Material examined: Holotype, female, FSL 391925 (fig. 14) from Aix. Paratype, female, FSL 391926. Paratype, female, MNHN B 24519 from Céreste. Female, attributed by Heer to Bibiopsis funebris Heer (ETHZ 164). 
Localities: Aix-en-Provence, Céreste.

Other material (MNHN): Males: Aix 779, 884, 974, 1085, P26. Females: Aix 332, 428, 645, 752, 799 , 890, 926, 965, 1013, 1061, 1322, 1337, 1347, 1404. (RGL) Céreste 662. Isolated wing: Céreste 192. Female, labeled 164 (ETHZ, fig. 15).

Diagnosis: The species is easily recognized by the long, horizontal vein $\mathrm{R}_{2+3}$, which is sharply bent basally and a bit sinuous in the middle. It is the only species in the French Oligocene material with a typical Penthetria wing venation. Specimens with preserved pigmentation are entirely dark with blackish-fumose wings.

\section{Redescription:}

Male: Total length $8.2-12.0 \mathrm{~mm}(\mathrm{~N}=5)$. The specimens are of a uniform, brown colour, probably not original colour.

Head: Length 0.96-1.30 mm ( $=5)$, width 1.03-1.41 mm ( $=4)$. Antenna robust, segments not possible to count.

Thorax: Length 2.2-2.9 mm ( $=5)$, width $1.7 \mathrm{~mm}(\mathrm{~N}=1$, smallest specimen).

Wing (fig. 102): Length 6.7-9.1 mm ( $=4)$, width 2.2-2.9 mm ( $=3)$, length/width $=2.98-3.30$ $(\mathrm{N}=3)$. No pigment preserved in membrane, $\mathrm{R}$-veins brown, posterior veins colourless. Costa extends to apex of $\mathrm{R}_{4+5}$. Humeral vein present, Subcosta terminating at origin of $\mathrm{R}_{2+3}$. Pterostigma oval, brown. $\mathrm{R}_{2+3}$ long, originating at base of pterostigma, bent in a right angle near base. Wing vein measurements in Table 1 .

Legs: Slender, first tarsomere of hind leg slightly swollen and spindle-shaped. Fore femur $2.2 \mathrm{~mm}$ $(\mathrm{N}=1)$, fore tibia $2.2-2.3 \mathrm{~mm}(\mathrm{~N}=2)$, hind femur $2.7-3.5 \mathrm{~mm}(\mathrm{~N}=3)$, hind tibia $2.5-3.8 \mathrm{~mm}(\mathrm{~N}=3)$.

Abdomen: Length 5.4-8.0 mm (N=5), width 1.1-1.6 mm ( $\mathrm{N}=2)$. Cylindrical.

Terminalia (fig. 101): Gonostylus digitiform, curved, pointed. Epandrium wide, dome-shaped. Hypopygium width $0.83-1.15 \mathrm{~mm}(\mathrm{~N}=4)$, gonostylus length $0.43 \mathrm{~mm}(\mathrm{~N}=1)$.

Female: Total length $7.5-12.0 \mathrm{~mm}(\mathrm{~N}=16)$. Holotype and one paratype very dark, probably the original colour. Most of the other specimens have little or no pigment preserved.

Head (fig. 99): Length $0.77-1.16 \mathrm{~mm}(\mathrm{~N}=11)$, width 0.82-1.28 mm ( $=17)$. Rounded. Complex eye medium-sized, slightly protruding. Ocellar triangle prominent but not very tall. Antenna robust, flagellum probably 8 -segmented.

Thorax: Length 1.9-3.1 mm (N=16), width 1.7-2.6 mm (N=15). Sulci prominent. Haltere dark brown.

Wing (figs. 98, 100): Length 7.0-10.5 mm ( $=18)$, width 2.7-3.9 mm ( $=16)$, length/width $=2.44$ $3.00(\mathrm{~N}=14)$. Blackish fumose, $\mathrm{R}$-veins dark, posterior veins concolourous with membrane. Costa terminates at apex of $\mathrm{R}_{4+5}$. Humeral vein present, Subcosta terminating at or slightly beyond origin of $\mathrm{R}_{2+3}$. Pterostigma oval, black. CuA-veins conspicuously strong. Vein $\mathrm{R}_{2+3}$ long and sinuous, basally vertical, bent $90^{\circ}$ and then running parallel to $\mathrm{R}_{4+5}$ before bending toward costa in distal half. Relative length of stem and fork of $\mathrm{CuA}$ apparently quite variable. $\mathrm{CuA}_{2}$ apically curved basad. Wing vein measurements in Table 1. 
Legs: Slender, covered with short erect dark pile. Length of fore femur 2.0-2.6 $\mathrm{mm}(\mathrm{N}=5)$, fore tibia $1.8-2.6 \mathrm{~mm}(\mathrm{~N}=6)$, hind femur 2.1-3.1 $\mathrm{mm}(\mathrm{N}=5)$, hind tibia 1.9-3.5 $\mathrm{mm}(\mathrm{N}=6)$.

Abdomen: Length 5.1-8.3 mm ( $\mathrm{N}=14)$, width 1.9-2.8 mm (N=13). Cylindrical.

Terminalia: Three spermathecae present. Cerci two-segmented, small and slender.

\section{Penthetria subterranea (Théobald, 1937) comb.n. (Figs. 18, 103-106)}

Plecia subterranea Théobald, 1937: 233.

Plecia bigoti Brongniart, 1879 - unpublished manuscript name in a collection of EM

Plecia gracilis Brongniart, 1879 - presumably an unpublished cabinet name in a collection of EM

Material examined: Holotype (male) NMB F1404. Paratypes (males) NMB F1414/1, F1414/2.

Additional material (2 males 11 females): EM 70002, 70009, 70010, 70011, 70013, 70015, 70017, 70201 (A 10.9), 70202 (A 10.13), 70203 (A 10.70), 70204 (A 10.93) (all in Coll. Coquand, EM, stored in FSL)

Localities: Kleinkembs, Aix-en-Provence.

Diagnosis: A medium-sized Penthetria (10-11 mm), male wing yellowish brown fumose. Vein $\mathrm{R}_{2+3}$ nearly straight. Male gonostylus digitiform, apically blunt.

\section{Redescription:}

Male: Total length $8.5-11.0 \mathrm{~mm}(\mathrm{~N}=7)$. Brown.

Head: Length $1.0-1.2 \mathrm{~mm}$, width $1.1-1.4 \mathrm{~mm}(\mathrm{~N}=4)$. Relatively small, broadly oval. Complex eye dichoptic? Flagellum 8-segmented, length $0.5 \mathrm{~mm}(\mathrm{~N}=1)$.

Thorax: Length 2.3-2.8 mm (N=7), width 1.8-2.0 mm (N=2). Black, convex. Sulci not particularly deep and conspicuous. Pile not conspicuous. Haltere brown.

Wing (fig. 103): Length 6.7-8.9 mm (N=5), width 2.5-2.9 mm ( $=4)$, length/width $=2.89-3.22$ $(\mathrm{N}=3)$. Yellowish fumose, rather narrow. Pterostigma small, pale brown. R-veins brown, posterior veins colourless. Costa extends to more than half-way between apices of $\mathrm{R}_{4+5}$ and $\mathrm{M}_{1}$. Subcosta long and prominent, humeral vein present. $\mathrm{R}_{2+3}$ oblique, nearly straight. Wing vein measurements in Table 1 .

Legs: Slender, yellowish, tarsi and apex of tibiae darker. Length of fore femur $1.9 \mathrm{~mm}(\mathrm{~N}=1)$, fore tibia 2.1-3.6 $\mathrm{mm}(\mathrm{N}=3)$, hind femur 2.4-3.7 $\mathrm{mm}(\mathrm{N}=3)$, hind tibia 2.6-3.6 $\mathrm{mm}(\mathrm{N}=2)$, hind first tarsomere $1.1 \mathrm{~mm}(\mathrm{~N}=1)$.

Abdomen: Length 5.4-9.4 mm ( $\mathrm{N}=7)$, width 1.2-1.5 mm (N=6). Long, slender, cylindrical. Pile not conspicuous. 
Terminalia (figs. 104-105): Hypopygium width 0.7-1.4 mm (N=6). Epandrium apparently undivided with slightly convex posterior edge. Gonostylus $0.41-0.62 \mathrm{~mm}$ long $(\mathrm{N}=4)$, digitiform, curved, apically blunt.

Female: Total length 6.0-9.6 mm (N=9)

Head: Length $0.59-0.80 \mathrm{~mm}(\mathrm{~N}=6)$, width $0.71-0.94 \mathrm{~mm}(\mathrm{~N}=5)$. Black. Antenna robust, flagellum $0.47-0.57 \mathrm{~mm}$ long $(\mathrm{N}=2), 8$-segmented?

Thorax: Length 1.8-2.5 mm (N=11), width 1.6-1.9 mm ( $=4)$. Dark yellowish-brown. Haltere pale brownish.

Wing (fig. 106): Length 5.8-8.2 mm ( $=10)$, width 2.2-3.2 mm ( $\mathrm{N}=7)$, length/width $=2.57-2.99$ $(\mathrm{N}=7)$. Hyaline to light brownish fumose, veins light brown. Venation as in male except that $\mathrm{R}_{2+3}$ is shorter and steeper. Wing vein measurements in Table 1.

Legs: Scarcely preserved in the specimens at hand. Yellowish-brown. Length of fore tibia 1.8-2.0 mm $(\mathrm{N}=2)$.

Abdomen: Length 3.7-6.4 mm ( $\mathrm{N}=10$, obviously shrunk in some specimens), width 1.5-1.9 mm $(\mathrm{N}=4)$. Brown.

Terminalia: Cercus rather long, two-segmented, terminal segment slender.

\section{Genus Plecia Wiedemann, 1828}

In the $19^{\text {th }}$ Century, fossil Plecia species were mostly placed in the genus Protomyia Heer, though many Penthetria species were also included in this genus.

\section{Plecia assonensis Gentilini, 1993 (Figs. 19, 107-109)}

Plecia assonensis Gentilini 1993: 161.

Possibly a synonym of Plecia livida, but the pointed gonostylus might be diagnostic together with the straight, oblique vein $\mathrm{R}_{2+3}$ and the indistinctive pterostigma.

Material examined: (RGL) Dauphin 33, Bois d'Asson 34, Bois d'Asson 81

\section{Localities: Bois d'Asson}

Diagnosis: A small Plecia (wing length approximately $5 \mathrm{~mm}$ ). Antennal flagellum 9-segmented. Vein $\mathrm{R}_{2+3}$ straight and oblique. Male gonostylus stout digitiform.

\section{Redescription:}

Male: Total length 5.0-8.5 $\mathrm{mm}(\mathrm{N}=2$, the former specimen obviously shrunk) 
Head: Length $0.81 \mathrm{~mm}(\mathrm{~N}=1)$, width $0.86-1.21 \mathrm{~mm}(\mathrm{~N}=2)$. Antennal flagellum 9-segmented according to Gentilini (1993: 161), not possible to see in the specimens at hand.

Thorax: Length 1.7-2.9 mm ( $=3)$, width 1.7-2.2 mm ( $\mathrm{N}=2)$. Brown. Haltere pale.

Wing (fig. 107): Length 5.1-5.7 mm (N=3), width 1.7-2.5 mm ( $=2)$, length/width $=2.31-3.01$ $(\mathrm{N}=2)$. Pale brownish, almost hyaline, veins brown, slender, $\mathrm{R}$-veins not notably stronger than posterior veins. Costa extends to a little beyond apex of $\mathrm{R}_{4+5}$. Subcosta fine, terminates approximately at fork of $\mathrm{M}$, humeral vein present. Vein $\mathrm{R}_{2+3}$ short, nearly straight and oblique, $\mathrm{R}_{4+5}$ slightly curved. Pterostigma small, light brown, indistinctive. Wing vein measurements in Table 2.

Legs: Light brownish, getting darker apicad. Hind femur apparently pale with darker apex.

Abdomen: Length 2.9-5.8 mm ( $\mathrm{N}=3)$, width 0.9-1.4 mm ( $\mathrm{N}=3)$. Brown, relatively broad.

Terminalia (figs. 108-109): Hypopygium 0.70-0.77 mm (N=3) wide, broad and robust. Gonostyli $0.35-0.46 \mathrm{~mm}(\mathrm{~N}=3)$, short, robust, apically pointed. Epandrium mesally with a shallow, $\mathrm{V}$-shaped cleft.

Female unknown.

\section{Plecia bucklandi (Heer, 1849) (Figs. 20-24, 110-113)}

Protomyia bucklandi Heer, 1849: 238-239.

Protomyia elegans Heer, 1856: 36. Syn.n.

Protomyia gracilis Heer, 1856: 36. Syn.n.

Bibio edwardsii Oustalet, 1870: 130 Syn.n.

Penthetria edwardsii (Oustalet, 1870)

Plecia edwardsii (Oustalet, 1870); Brongniart, 1878: XLVIII.

Plecia dilatativentris Théobald, 1937: 338-339. Syn.n.

The species was described from Radoboj and Aix-en-Provence. Heer in his original description did not fix any holotype, but the description was made with reference to Buckland's (1837, Pl. 46) illustration of a specimen from Aix-en-Provence, so this should be regarded as the type. We could not find this specimen in NHM. The name has generally been spelled bucklandi as the species was named after the Rev. William Buckland. However, the somewhat awkward form buklandi was used in the heading of the original description (Heer 1849 p. 238). The form bucklandi was however, used in the legends to the plates (Heer 1849, Pl. XVI, Fig. 22) and in all subsequent treatises.

Material examined: Two female specimens of Protomyia bucklandi from Aix in ETHZ, not among those illustrated by Heer (1849, Pl. XVI, Fig. 22), but labeled in Heer's handwriting. Figured specimen (female) FSL 391930 (A 25). Syntype (female) of Protomyia elegans (NHMUK In 39832). Syntype (female) of Protomyia gracilis (NHMUK In 38765). One specimen labeled as Protomyia gracilis in ETHZ, not the one illustrated by Heer but the name is written in Heer's handwriting. Syntypes (3 females) of Bibio edwardsii (MNHN R 06671, R 06669). Holotype (female) of Plecia dilatativentris FSL 391924. Paratypes (2 females) of Plecia dilatativentris MNHN B 24418. Additional material (6 females) FSL 391928, 391931-391935. 
Localities: Aix-en-Provence, Corent.

Diagnosis: A medium-large $(7.5-10.5 \mathrm{~mm})$ Plecia, body and wings dark brown, probably black in life. Antennal flagellum probably 8 -segmented. Fore femorae apparently pale brown.

\section{Redescription:}

Male unknown

Female Total length $7.5-10.5 \mathrm{~mm}(\mathrm{~N}=11)$, dark brown, presumably black in life.

Head (figs. 112-113): Length 0.87-1.14 mm ( $=5)$, width 0.96-1.35 mm (N=5). Rather short and broad, complex eye a little prominent. Antenna rather short, flagellum probably 8-segmented, cylindrical, 0.57-0.71 $\mathrm{mm}$ long $(\mathrm{N}=4)$.

Thorax: Length 2.2-3.0 mm (N=9), width 1.7-2.6 mm ( $\mathrm{N}=4)$. Black. Haltere brown.

Wing (figs. 110-111): Length 7.0-9.2 mm ( $\mathrm{N}=10)$, width $2.5-3.7 \mathrm{~mm}(\mathrm{~N}=8)$, length/width $=2.45-$ $3.25(\mathrm{~N}=8)$. Blackish fumose, veins brownish black, prominent throughout wing. Pterostigma indistinctive due to generally dark membrane. Subcosta prominent, long, humeral vein present. Costa extending to apex of $R_{4+5}$. $R_{2+3}$ a little curved, oblique. Wing vein measurements in Table 2 .

Legs: relatively short, slender, femorae brownish, tibiae and tarsi black. Length of fore femur $1.7 \mathrm{~mm}$ $(\mathrm{N}=1)$, fore tibia $1.6-2.2 \mathrm{~mm}(\mathrm{~N}=5)$.

Abdomen: Length 5.2-7.7 mm (N=8), width 1.8-2.8 mm (N=6), black, cylindrical.

Terminalia: cerci short and indistinct.

\section{Plecia conica Théobald, 1937 (Figs. 29-30, 114-118)}

Plecia conica Théobald, 1937: 232.

Plecia haennii Gentilini, 1993: 164. Syn.n.

Material: Holotype (male) of Plecia conica NMB F1398/1, paratype (male) of Plecia conica NMB F1398/2. Non-type material (3 males) NMB F2090, (RGL) Dauphin 12, Saint-Maime 23

Plecia conica may quite possibly be the male of Plecia foersteri (next species).

Localities: Bois d'Asson, Kleinkembs

Diagnosis: A small Plecia (total length 5-7 mm). Antennal flagellum 8-segmented. Male epandrium dome-shaped, gonostylus very robust, apically expanded.

\section{Redescription:}


Male (figs. 29-30, 115-116): Total length 5.0-7.0 mm (N=4).

Head: Length $0.85-0.90 \mathrm{~mm}(\mathrm{~N}=2)$, width $0.93 \mathrm{~mm}(\mathrm{~N}=1)$. Antennal flagellum 8-segmented. Ocellar tubercle rather small.

Thorax: Length 1.7-2.2 mm (N=3), width $1.5 \mathrm{~mm}(\mathrm{~N}=1)$. Black. Haltere yellowish-brown.

Wing (fig. 114): Length 4.8-5.0 mm ( $=3)$, width $2.0-2.1 \mathrm{~mm}(\mathrm{~N}=2)$, length/width $=2.39-2.50$ $(\mathrm{N}=2)$, elliptical. Light brownish fumose, veins fine and light brown. Pterostigma very indistinctive. $\mathrm{R}_{2+3}$ straight and oblique. Apex of $\mathrm{R}_{4+5}$ slightly curved.

Legs: Slender, pale brown. Length of fore tibia $1.6 \mathrm{~mm}(\mathrm{~N}=1)$, hind femur $1.9 \mathrm{~mm}(\mathrm{~N}=1)$, hind tibia $2.0 \mathrm{~mm}(\mathrm{~N}=1)$, hind first tarsomere $0.7 \mathrm{~mm}(\mathrm{~N}=1)$.

Abdomen: Length 3.3-4.6 mm (N=3). Brown, conical.

Terminalia (figs. 117-118): Hypopygium width $0.57-0.72 \mathrm{~mm}(\mathrm{~N}=2)$. Gonostylus $0.26-0.32 \mathrm{~mm}$ long $(\mathrm{N}=2)$, strong, clavate, thickened apically. Epandrium mesally with a shallow indentation.

Female unknown

\section{Plecia foersteri Théobald, 1937 (Figs. 25-28, 119-122)}

Plecia Försteri Théobald, 1937: 136.

Plecia splendida Théobald, 1937: 137 Syn.n.

Plecia oviformis Théobald, 1937: 229. Syn.n.

Plecia rubescens (Oustalet, 1870) sensu Théobald 1937: 230.

Material examined: Holotype (female) of Bibio foersteri NMB F1390. 4 paratypes (females) of Bibio foersteri NMB F1391-1394. Lectotype (female, herein designated) of Plecia splendida NMB F1396. Paratype (female) of Plecia spendida NMB F1397. Paratype (female) of Plecia splendida MNHN B 23563. Holotype (female) of Plecia oviformis NMB F1384.

Théobald (1937: 137 and 231) described Plecia splendida with two "holotypes" (should be syntypes), one from Kleinkembs and the other one from Célas. We have studied the former but did not succeed in locating the latter, which may be lost. The holotype of Protomyia rubescens is not recognizable, as was also commented on by Théobald (1937). He therefore (presumably) designated a "hypotypoide" for "Plecia cf. rubescens" (NHMB F1395/1), this specimen has no type status but is useful in clarifying Théobald's concept of the species.

Additional material (28 females): NMB F1395/1, F1410, F1411/1-22, F1412, F2048, F2175, F2223, F2368.

Localities: Kleinkembs, Ronzon, Célas. 
Diagnosis: A small-medium sized Plecia (wing length 4.9-7.8 mm). Antennal flagellum 8-segmented. Thorax black, abdomen brown, wing light brownish fumose.

\section{Redescription:}

Male unknown. There is one male specimen (NHMB F 2090) in NHMB labelled as Plecia cf. rubescens, but it is highly uncertain if this is conspecific with the females.

Female: Total length $6.2-8.5 \mathrm{~mm}(\mathrm{~N}=23)$.

Head (figs. 120, 122): Length 0.59-0.78 mm ( $=12)$, width 0.63-0.96 mm ( $\mathrm{N}=15)$. Rather short and broad, oval, complex eye prominent. Antenna length $0.41-0.62 \mathrm{~mm}(\mathrm{~N}=8)$, rather short and thick, flagellum 8-segmented. Ocellar tubercle small.

Thorax: Length 1.7-2.7 mm (N=27), width 1.4-2.0 mm (N=16). Black. Sulci deep and distinctive. Haltere brown.

Wing (fig. 119): Length 5.4-8.0 mm ( $=26)$, width 1.9-3.2 mm ( $=23)$, length/width $=2.10-2.87$ $(\mathrm{N}=23)$. Light brownish fumose, veins brown. Subcosta long and distinctive, humeral vein present. Costa extending to apex of $\mathrm{R}_{4+5}$. $\mathrm{R}_{2+3}$ slightly curved, oblique. Fork of $\mathrm{M}$ rounded. Pterostigma indistinctive. Wing vein measurements in Table 2.

Legs: Brown, slender.

Abdomen: Brown, spindle-shaped.

Terminalia: Cerci short and indistinctive.

\section{Plecia inflata Oustalet, 1870 (Figs. 31, 123-125)}

Plecia inflata Oustalet, 1870: 142.

Material examined: Holotype (female) NMB F1495.

Additional material (5 females) NMB F2152, F2210, F2215, F2344, F2345.

Localities: Corent, Kleinkembs.

Diagnosis: A medium-large Plecia (total length 8.7-10.7 mm). Antennal flagellum probably 8segmented. The species has highly distinctive female terminalia with a pair of strong, hook-shaped appendages on the last sternite.

\section{Redescription:}

Male unknown. 
Female: Total length $8.7-10.7 \mathrm{~mm}(\mathrm{~N}=6)$

Head (fig. 124): Length $1.02 \mathrm{~mm}(\mathrm{~N}=1)$, width 1.01-1.07 mm ( $\mathrm{N}=2)$. Black. Antennal flagellum rather slender, probably 8 -segmented. Complex eye prominent. Ocellar tubercle low and indistinctive.

Thorax: Length 2.5-2.9 mm (N=6). Black. Haltere light brown.

Wing (fig. 123): Length 8.4-9.8 mm ( $\mathrm{N}=3)$, width $3.4 \mathrm{~mm}(\mathrm{~N}=1)$, length/width $=2.53(\mathrm{~N}=1)$. Light brown fumose, veins brown. Costa extending to apex of $\mathrm{R}_{4+5}$. Subcosta long and distinctive, humeral vein present. Pterostigma indistinctive. $\mathrm{R}_{2+3}$ rather long, curved. CuA with a long stem and short, rounded fork.

Legs: Dark brown, rather short, slender throughout. Fore femur 2.2-2.3 mm (N=2), fore tibia 2.2-2.3 $\mathrm{mm}(\mathrm{N}=2)$, hind femur 2.6-2.8 $\mathrm{mm}(\mathrm{N}=3)$, hind tibia $2.7 \mathrm{~mm}(\mathrm{~N}=2)$.

Abdomen: Length 5.9-7.7 mm (N=6). Brown, cylindrical.

Terminalia (fig. 125): Dark brown. Last sternite much darker and heavier sclerotized than preceeding sternites. Last sternite with a pair of strong, hook-shaped appendages.

\section{Plecia lapidaria (Heyden \& Heyden, 1865) (Figs. 32-33, 126-128)}

Protomyia lapidaria Heyden \& Heyden, 1865: 25.

Plecia lapidaria (Heyden \& Heyden, 1865) Théobald 1937: 231-232.

Plecia Bayleana Brongniart, 1879 - presumably an unpublished cabinet name in collection of EM

Plecia Heeri Brongniart, 1879 - presumably an unpublished cabinet name in collection of EM

Material examined: Syntype (female) of Protomyia lapidaria NHMUK In 58813. 15 females, NMB F1496, F1530, F1565, F1600, F2044, F2059, F2060, F2089, F2103, F2131, F2177, F2242, F2287, F2389, F2406. 1 female, Aix-en-Provence FSL 391927. 5 females, EM 70003, 70004, 70005, 70012, 70205 (A 10.95) (Coll. Coquand, stored at FSL).

Localities: Kleinkembs, Aix-en-Provence. The type is from Rott, Germany.

Diagnosis: A rather small Plecia, total length 4.8-6.5 mm. Antennal flagellum rather long, 9segmented. It can be distinguished from Plecia livida on the longer antenna and by the wider wing which is darkened anteriorly.

\section{Redescription:}

Male unknown

Female: Total length 4.8-6.8 mm (N=14).

Head (fig. 127): Length $0.55-0.85 \mathrm{~mm}(\mathrm{~N}=8)$, width $0.74-1.12 \mathrm{~mm}(\mathrm{~N}=12)$. Black, rather wide, rounded. Antennal flagellum rather long, cylindrical, 9-segmented. Ocellar triangle prominent.

Thorax: Length 1.5-2.1 mm (N=15), width 1.3-1.6 mm (N=6). Black. Sulci deep. Haltere brown. 
Wing (fig. 126): Length 4.9-6.7 mm ( $\mathrm{N}=7)$, width 2.0-3.0 mm ( $=5)$, length/width $=2.17-2.47$ $(\mathrm{N}=5)$. Oval, comparatively broad, anterior margin conspicuously curved. Light brown fumose, anterior part darkened, veins brown throughout. Costa extends almost to apex of $\mathrm{M}_{1}$. Pterostigma brown, does not extend below $\mathrm{R}_{1}$, conspicuous. $\mathrm{R}_{2+3}$ with a kink in basal fourth, otherwise nearly straight, oblique. Crossvein $\mathrm{M}-\mathrm{Cu}$ some distance from base of $\mathrm{CuA}_{1}$. Wing vein measurements in Table 2 .

Legs: Dark brown, slender. Hind tibia $1.9 \mathrm{~mm}(\mathrm{~N}=1)$.

Abdomen: Length 3.2-4.6 mm ( $\mathrm{N}=14)$, width 1.5-1.8 mm (N=7). Blackish brown.

Terminalia (fig. 128): Cerci small, rounded triangular.

\section{Plecia larguensis Gentilini, 1993 (Figs. 34-35, 129-131)}

Plecia larguensis Gentilini, 1993: 162

Material (tentative) (RGL) Bois d'Asson 5, Bois d'Asson 7, Dauphin 30, Bois d'Asson 47, Dauphin 182.

Localities: Bois d'Asson

Diagnosis: A rather small species $(6-7.5 \mathrm{~mm})$. The species can be recognized on the hooked, pointed gonostyli and the epandrium bearing three long projections, further on the very short, nearly vertical vein $\mathrm{R}_{2+3}$ and the large, light brown pterostigma. The female is unknown, most likely rather similar to Plecia livida.

\section{Redescription:}

Male: Total length 6.0-7.5 mm (N=5).

Head (fig. 130): Length 0.86-1.02 mm (N=3), width 0.84-1.02 mm ( $=3)$. Antennal flagellum cylindrical, 9- or 10-segmented. Ocellar tubercle rather tall, rounded. Palp robust. Rostrum a little elongated, about two thirds as long as complex eye.

Thorax: Length 1.6-1.9 mm (N=5). Dark reddish brown.

Wing (fig. 129): Length 4.9-5.4 mm (N=5), width 1.9-2.1 mm ( $\mathrm{N}=5)$, length/width $=2.42-2.65$ $(\mathrm{N}=5)$. Light brownish fumose, pterostigma large, oval and light brown. Veins brown throughout, $\mathrm{R}-$ veins scarcely darker than posterior veins. Costa extends almost half-way between apices of $R_{4+5}$ and $M_{1}$. Subcosta fine and indistinctive. $R_{2+3}$ short, nearly vertical, $R_{4+5}$ nearly straight. Wing vein measurements in Table 2.

Legs: Reddish brown, femorae paler in basal half. Slender throughout. Fore femur 1.6-2.2 mm (N=4), fore tibia 1.9-2.1 $\mathrm{mm}(\mathrm{N}=2)$, hind femur $2.2 \mathrm{~mm}(\mathrm{~N}=2)$, hind tibia $2.2-2.3 \mathrm{~mm}(\mathrm{~N}=2)$, hind first tarsomere $0.8 \mathrm{~mm}(\mathrm{~N}=1)$.

Abdomen: Length 3.9-4.9 mm (N=5). Brown, slender. 
Terminalia (fig. 131): Hypopygium width 0.70-0.95 mm ( $\mathrm{N}=4)$. Epandrium small, with three long, digitiform projections. Gonostylus $0.37-0.49 \mathrm{~mm}$ long $(\mathrm{N}=4)$, apically curved and sharply pointed.

Female unknown. The material from Bois d'Asson contains numerous females which are seemingly rather homogenous morphologically. This material presumably contains females of all the four nominal species named by Gentilini (1993), as well as Plecia livida.

\section{Plecia livida (Heer, 1856) (Figs. 36-37, 39, 41, 132-143)}

Protomyia livida Heer, 1856: 35

Protomyia brevipennis Heer, 1856: 35 Syn.n.

Plecia livida (Heer, 1856) Théobald 1937: 339.

Plecia brevithoracis Théobald, 1937: 409 Syn.n.

Plecia cerestensis Théobald, 1937: 409 Syn.n.

Plecia constricta Théobald, 1937: 342 Syn.n.

Plecia flichei Théobald, 1937: 406 Syn.n.

Plecia fusciventris Théobald, 1937: 407 Syn.n.

Plecia goreti Théobald, 1937: 409 Syn.n.

Plecia maculitarsis Théobald, 1937: 411 Syn.n.

Plecia maculiventris Théobald, 1937: 411 Syn.n.

\section{Material:}

Lectotype of Protomyia livida (female, herein designated), NHMUK In 39854. This is the specimen illustrated by Curtis (1829), Heer described the specimen with reference to this illustration. Paralectotype (female, herein designated) from Aix-en-Provence (ETHZ, Aix 120). The ETHZ specimen is labelled livida in Heer's handwriting (partly obliterated).

Holotype (female) of Plecia goreti MNHN B 24516. Paratype (female) of Plecia guineri MNHN B 24415. Holotype (male) of Plecia maculiventris MNHN B 24523. Holotype (male) of Plecia brevithoracis EEFN (Coll. Fliche) F 298. Holotype (female) of Plecia fusciventris EEFN (Coll. Fliche) F 285. Holotype (female) of Plecia flichei EEFN (Coll. Fliche) F 310. Paratype (female) of Plecia flichei EEFN (Coll. Fliche) F 178. "Type" (male, exact status unclear) of Protomyia brevipennis MNHN B 24413. Syntype (male) of Plecia brevipennis NHM In 39830.

Localities: Céreste, Aix-en-Provence, Bois d'Asson, Barbeiras, Chadrat, Vitrolles

Of the approximately 2570 numbered specimens from Céreste in RGL, $80 \%$ probably belong to this species, though most are too poorly preserved for a certain species identification.

Diagnosis: A medium-sized Plecia, body length 5.5-10 $\mathrm{mm}$ (much of the variation due to postmortem swelling), wing length 5-8.5 mm. Head with rostrum not at all produced. Flagellum short and 9 -segmented in female, slender and 8-segmented in male. Femorae pale with dark tips, more distal leg segments usually dark. Wings light brownish in most specimens (other colours most likely due to postmortem chemical changes), veins brown, distinctive throughout the wing. Abdomen yellowish-brown. 


\section{Redescription:}

Male: Total length 5.5-10.5 mm ( $\mathrm{N}=100)$.

Head (fig. 134): Length 0.92-1.25 mm ( $\mathrm{N}=22)$, width $1.00-1.45 \mathrm{~mm}(\mathrm{~N}=22)$. Brown. Complex eye seemingly naked. Antenna cylindrical, flagellum 8-segmented. Flagellum length 0.6-0.7 mm (N=2).

Thorax: Length 1.6-2.5 mm (N=55), width 1.3-1.6 mm ( $=4)$. Brown. Haltere brown.

Wing (fig. 132): Length 5.0-6.8 mm ( $=29)$, width $1.8-2.6 \mathrm{~mm}(\mathrm{~N}=29)$, length/width $=2.27-2.95$ $(\mathrm{N}=29)$. Light brownish. Triangular with $\mathrm{R}_{2+3}$ short and curved. Veins distinctive and brown throughout wing. Pterostigma light brown, indistinctive. Wing vein measurements in Table 2.

Legs: Light brown. Fore tibia length 1.9-2.7 mm ( $\mathrm{N}=19)$, fore first tarsomere 0.9-1.3 mm $(\mathrm{N}=10)$, hind tibia $2.8-2.9 \mathrm{~mm}(\mathrm{~N}=2)$.

Abdomen: Yellowish-brown, slender.

Terminalia (figs. 136-141): Epandrium dome-shaped. Gonostyli approximately $0.4 \mathrm{~mm}$ long, spoonshaped, appearance very much depending on perspective. Aedeagus and associated structure as in fig. 141.

Female: Total length 5.0-10.4 mm (N=142).

Head (fig. 135): Length 0.60-0.75 mm ( $=4)$, width 0.77-1.10 mm ( $=3)$. Brown. Complex eye relatively large and flat. Antenna cylindrical, flagellum 9-segmented.

Thorax: Length 1.4-2.4 mm (N=53). Brown. Haltere light brown.

Wing (fig. 133): Length 5.1-8.9 mm ( $\mathrm{N}=141)$, width $2.1-3.9 \mathrm{~mm}(\mathrm{~N}=40)$, length/width= 2.13-2.80 $(\mathrm{N}=40)$. Oval, yellowish-brown, veins brown and relatively slender. Costa extends to half-way between apices of $R_{4+5}$ and $M_{1}$. Subcosta long, straight, running close to $R_{1}$ for most of its length. $R_{2+3}$ oblique, slightly curved, longer than in male. Pterostigma indistinctive. Crossvein $\mathrm{M}-\mathrm{Cu}$ near base of $\mathrm{CuA}_{1}$. Wing vein measurements in Table 2.

Legs: Light brownish, slender. Fore tibia 1.6-2.1 mm (N=8).

Abdomen: Cylindrical, yellowish-brown.

Terminalia (figs. 142-143): Cerci small, apically knob-shaped.

\section{Plecia lygaeoides (Heer, 1849) (Figs. 38, 40, 42-44, 144-148)}

Protomyia lygaeoides Heer, 1849: 232-233.

Bibio lignarius Germar, 1837, sensu Unger, 1842, misidentification, pro parte.

Protomyia gracilenta Heyden \& Heyden, 1865: 28. Syn.n.

Bibio detractus Theobald, 1937: 336 Syn.n.

Plecia guinieri Théobald, 1937: 408 Syn.n.

Plecia acutiventris Théobald, 1937: 410 Syn.n.

Protomyia lygaeoides was described from Radoboj and later noted from Aix-en-Provence by Heer (1856). The type is in Landesmuseum Joanneum, Graz and an additional specimen from Aix-en- 
Provence is in ETHZ. Protomyia gracilenta was decribed from Rott, Plecia acutiventris and Bibio detractus from Aix-en-Provence.

Material examined: Female identified as Bibio lignarius sensu Unger SLJG 259. Female (NHMUK In 39822). Holotype (male) of Protomyia gracilenta NHMUK In 58805. Cotypes ( 2 males 1 female) of Plecia guinieri EEFN (coll. Fliche) F 190. Cotype (female) of Plecia guinieri EEFN (Coll. Fliche) F 239. Holotype (male) of Plecia acutiventris FSL 391937 (A84), EEFN F 169 (status of both specimens given as "holotype" in figure legends (Pl. XXII Fig. 9 and Pl. XXIX Fig. 14) by Théobald (1937)). Holotype (male) of Bibio detractus FSL 391921 (A 21). Paratype (male) FSL 391922 (A 29).

Other material: 9 males EEFN (Coll. Fliche) F 176, F 182, F 187, F 188, F 200, F 207, F 268, F 302, F 320. Female (FSL 391902), Chadrat.

Localities: Aix-en-Provence, Chadrat (Radoboj, Rott)

Diagnosis: A medium-sized, dark Plecia. The wings are blackish fumose with all veins black, Pterostigma oval and distinctive, vein $\mathrm{R}_{2+3}$ straight and oblique. Male gonostylus long and slender.

\section{Redescription:}

Male: Total length 7.0-9.5 mm ( $\mathrm{N}=14)$.

Head (fig. 145): Length 1.0-1.1 mm (N=34), width 1.1-1.3 mm (N=3). Flagellum 0.48 mm long, 8segmented.

Thorax: Length 1.9-3.0 mm (N=7), width 1.8-2.1 mm (N=4). Entirely black.

Wing: Length 4.8-7.0 mm ( $\mathrm{N}=14)$, width 2.0-2.5 mm ( $\mathrm{N}=5)$, length/width $=2.45-3.00(\mathrm{~N}=5)$. Dark brownish fumose, all veins pigmented, dark brown. Pterostigma oval, dark brown, moderately distinctive. Vein $\mathrm{R}_{2+3}$ straight and oblique. Wing vein measurements in Table 2.

Legs: Dark brown to black, slender, tibiae conspicuously thin. Fore tibia 2.0-2.6 mm (N=4), hind femur $1.8 \mathrm{~mm}(\mathrm{~N}=1)$, hind tibia 2.1-2.9 $\mathrm{mm}(\mathrm{N}=4)$.

Abdomen: Length 4.6-5.3 mm (N=5), width 1.1-1.6 mm ( $=4)$, black, slightly conical.

Terminalia (fig. 147-148): Hypopygium width $0.79-0.80 \mathrm{~mm}(\mathrm{~N}=3)$, gonostylus length $0.39-0.53 \mathrm{~mm}$ $(\mathrm{N}=3)$. Gonostylus digitiform, rather narrow.

Female: Total length 8.0-11.4 mm (N=6), entirely black.

Head (fig. 146): Length 0.81-1.05 mm ( $=2)$, width 1.30-1.35 mm (N=2). Black. In dorsal view with prominent hind corners. Antenna relatively long and robust, flagellum 10-segmented. Flagellum length $0.74 \mathrm{~mm}(\mathrm{~N}=1)$. Palp strong.

Thorax: Length 2.0-3.1 mm (N=6), width 2.0-2.4 mm ( $\mathrm{N}=4)$. Black, haltere brown.

Wing (fig. 144): Length 6.2-9.8 mm ( $\mathrm{N}=7)$, width $2.5-3.6 \mathrm{~mm}(\mathrm{~N}=4)$, length/width $=2.43-2.55$ $(\mathrm{N}=4)$. Blackish fumose with blackish veins. $\mathrm{R}_{2+3}$ oblique. Wing vein measurements in Table 2. 
Legs: Black. Hind femur moderately clavate, otherwise slender. Length of fore femur $2.0 \mathrm{~mm}(\mathrm{~N}=1)$, fore tibia 1.7-2.9 $\mathrm{mm}(\mathrm{N}=4)$, hind femur $2.8 \mathrm{~mm}(\mathrm{~N}=1)$, hind tibia 2.8-3.2 $\mathrm{mm}(\mathrm{N}=2)$.

Abdomen: Length 5.7-8.7 mm ( $=6)$, width 2.1-3.3 mm (N=3). Black.

Terminalia: Cerci small and rounded.

\section{Plecia maimensis Gentilini, 1993 (Figs. 45-46, 149-153)}

Plecia maimensis Gentilini, 1993: 158.

We have not studied the type of this species. It is possibly a synonym of Plecia livida, but this is uncertain. Unique among fossil bibionids, this species was described from a male and female specimen in copula, thus the association of male and female is less tentative than in the other fossil species.

Material (tentative) Males: RGL Bois d'Asson 3, Bois d'Asson 24, Bois d'Asson 85, Bois d'Asson 90, Bois d'Asson 122, Bois d'Asson 139, Bois d'Asson 179.

Females: RGL Dauphin 1, Dauphin 2, Bois d'Asson 4, Dauphin 28, Bois d'Asson 70, Dauphin 132, Dauphin 144, Dauphin 172, Bois d'Asson 176.

Localities: Bois d'Asson

Diagnosis: The species is quite similar to Plecia livida, but the rounded lobe at the base of the gonostylus in the male and the slight kink in Rs at the furcation with $\mathrm{R}_{2+3}$ in the female may be diagnostic for the species.

\section{Redescription:}

Male: Total length $4.8-8.3 \mathrm{~mm}(\mathrm{~N}=7)$.

Head (fig. 150): Length $0.77-0.97 \mathrm{~mm}(\mathrm{~N}=3)$, width $1.01 \mathrm{~mm}(\mathrm{~N}=1)$. Dark brown, probably black in life. Antenna slender, flagellum 7- or 8-segmented. Rostrum slightly elongated, approximately half as long as complex eye. Ocellar triangle rather low, not very prominent. Palp robust.

Thorax: Length 1.5-2.4 mm (N=7), width $1.6 \mathrm{~mm}(\mathrm{~N}=1)$. Dark brown, probably black in life. Pile not conspicuous. Haltere pale.

Wing (fig. 149): Length 4.3-6.1 mm (N=7), width 1.6-2.4 mm ( $=7)$, length/width $=2.42-2.79$ $(\mathrm{N}=7)$. Light brownish, radial veins brown, more posterior veins concolourous with membrane.

Subcosta distinctive, straight. $\mathrm{R}_{2+3}$ short and curved. Costa extends to nearly half-way between apices of $R_{4+5}$ and $M_{1}$. $R_{4+5}$ apically moderately curved. Wing vein measurements in Table 3 .

Legs: Brown, slender but not particularly long. Tarsi slender. Metatibial spur dark brown, short, relatively strong. Length of fore femur $1.5 \mathrm{~mm}(\mathrm{~N}=1)$, fore tibia $1.3-1.8 \mathrm{~mm}(\mathrm{~N}=4)$, hind femur 1.6$2.2 \mathrm{~mm}(\mathrm{~N}=4)$, hind tibia $1.8-2.3 \mathrm{~mm}(\mathrm{~N}=6)$.

Abdomen: Length 3.1-5.9 mm (N=7). Brown, conical. 
Terminalia (fig. 152): Hypopygium width $0.75-0.85 \mathrm{~mm}(\mathrm{~N}=4)$. Dark brown. Epandrium domeshaped, apically with three short, knob-like points. Gonostylus 0.32-0.57 mm long ( $\mathrm{N}=6)$, slender, digitiform, slightly curved, basally with a rounded, knob-like dorsal lobe.

Female: Total length 7.0-9.5 mm (N=9)

Head (fig. 151): Length 0.81-0.85 mm (N=2), width 0.75-1.04 mm ( $=7)$. Dark brown, rounded, a little wider than long. Complex eye flat, about as long as temples. Antenna slender and pointed, flagellum 7- or 8-segmented.

Thorax: Length 1.9-2.9 mm (N=8), width 1.5-2.1 mm (N=6). Dark brown. Haltere light brown.

Wing: Length 5.9-8.1 mm (N=9), width 2.3-3.2 mm (N=9), length/width = 2.31-2.71 (N=9). Light brownish fumose, veins brown. Pterostigma light brown, rather indistinctive. Costa extends half-way between apices of $\mathrm{R}_{4+5}$ and $\mathrm{M}_{1}$. Rs with a little kink where $\mathrm{R}_{2+3}$ branches off. $\mathrm{R}_{2+3}$ moderately long, strongly curved basally. $\mathrm{R}_{4+5}$ moderately curved apically. Crossvein R-M vertical. Wing vein measurements in Table 3.

Legs: Dark brown.

Abdomen: Length 4.5-7.2 mm (N=9), width 1.5-2.4 mm (N=8). Reddish brown, robust.

Terminalia (fig. 153): Cerci large and prominent, two-segmented.

The sexual size dimorphism of this species was commented upon by Gentilini (1993). Such a size difference is possible due to the large variability in size in bibionids, and it is probably as common a trait among fossil bibionids as it is among recent ones. Caution is warranted when trying to associate male and female specimens since females may be considerably larger than conspecific males. This trend is not universal, however, since in many recent species females are smaller than males at least with respect to body length.

\section{Plecia morio (Heer, 1849) (Figs. 47-48, 154-159)}

Bibio morio Heer, 1849: 222

Protomyia collossea Heyden \& Heyden, 1865: 21.Syn.n.

Plecia collossea (Heyden \& Heyden, 1865) Statz, 1943: 14

Plecia lygaeoides (Heer, 1849) sensu Théobald, 1937

Bibio morio Heer is not to be confused with Bibio morio (Linnaeus, 1758) sensu Fabricius, 1787 (current name Hemipenthes morio (Linnaeus, 1758)). This species was, however, described in the genus Musca and not in Bibio, so Bibio morio Heer is not a primary junior homonym.

Material examined: 1 female (part and counterpart) NHMUK In 39831 and 39860. Females FSL 391929, FSL 391941. MNHN Aix 411, 970, 1295, 1386, 1442. Syntype (female) of Protomyia collossea NHMUK In 58789.

Male (tentatively associated) MNHN Aix 388, 885, 1398.

Localities: Aix-en-Provence. Also from Rott, Germany.

The species was described from Radoboj, but we did not find the type specimen in SLJG nor in NHMW. However, the specimen recorded by Heer (1856: 33) from Aix-en-Provence is easily 
recognizable on being considerably larger than all other specimens recorded by Heer from Aix. The specimens fit Heer's description well.

Diagnosis: A large (13-15 mm), dark Plecia species with dark wings which are darkened in the anterior half, at least in females. The species is easily separated from the other species from French Oligocene sediments by its size. The body proportions of the female are characteristic, with a very small head and large, cylindrical abdomen. Antennal flagellum is cylindrical and nine-segmented. Male: terminalia with long, slender and nearly straight gonostyli.

\section{Redescription:}

Male: Total length $13.5-14.0 \mathrm{~mm}(\mathrm{~N}=3)$.

Head (figs. 156-157): Length 1.68-1.72 mm (N=3), width 1.72-1.84 mm (N=3). Brown. Antenna relatively long and slender, flagellum probably 9-segmented. Interocular pile not apparent.

Thorax: Length 3.8-4.0 $\mathrm{mm}(\mathrm{N}=3)$, width $2.8-3.2 \mathrm{~mm}(\mathrm{~N}=3)$. Brown, probably black in life. Haltere pale.

Wing (fig. 154): Length 10.0-11.5 mm (N=3), width 3.5-4.0 mm (N=3), length/width $=2.77-2.90$ $(\mathrm{N}=3)$. Pigmentation of membrane and veins not preserved in any of the specimens at hand. Humeral vein and subcosta prominent, pterostigma indistinctive. $R_{2+3}$ evenly curved, apical part of $R_{4+5}$ rather straight. Fork of $\mathrm{M}$ relatively short. Wing vein measurements in Table 3.

Legs: Not preserved in the specimens at hand.

Abdomen: Length 8.5-9.2 mm ( $\mathrm{N}=3)$, width 1.6-1.8 mm ( $\mathrm{N}=3)$. Light brown, relatively long and slender. Pile not apparent in the specimens at hand.

Terminalia: Dark brown. Hypopygium width 1.5-1.6 mm (N=3). Epandrium posteriorly with wide, $\mathrm{U}-$ shaped indentation. Gonostylus $0.90 \mathrm{~mm}$ long $(\mathrm{N}=1)$, slender, nearly straight.

Female: Total length $14.0-16.2 \mathrm{~mm}(\mathrm{~N}=7)$.

Head (figs. 158-159): Length 1.14-1.45 mm ( $=4)$, width 1.22-1.64 mm ( $=4)$. Brown, probably black in life, rather small. Antenna cylindrical, slender, flagellum $1.11 \mathrm{~mm}$ long $(\mathrm{N}=1)$, 9-segmented. Complex eye medium-sized, oval. Ocellar triangle low and indistinctive.

Thorax: Length 3.0-4.1 mm (N=7), width 2.4-3.3 mm ( $=4)$. Dark brown, probably black in life. Pile not apparent in the specimens at hand.

Wing (fig. 155): Length 12.0-13.2 mm ( $\mathrm{N}=8)$, width 4.2-4.7 mm ( $\mathrm{N}=7)$, length/width $=2.66-3.09$ $(\mathrm{N}=7)$. Brown, anterior half somewhat darker. Veins brown, strong and distinctive. Humeral vein and subcosta prominent. Pterostigma indistinctive. $\mathrm{R}_{2+3}$ evenly curved, rather short, apical part of $\mathrm{R}_{4+5}$ nearly straight. Costa extends a little beyond apex of $\mathrm{R}_{4+5}$. Wing vein measurements in Table 3 .

Legs: Femorae and tibiae yellowish brown, tarsi dark brown, presumably black in life. Slender throughout, relatively short. Fore femur $3.1 \mathrm{~mm}(\mathrm{~N}=1)$, fore tibia $3.6 \mathrm{~mm}(\mathrm{~N}=1)$, hind tibia $4.6 \mathrm{~mm}$ $(\mathrm{N}=1)$.

Abdomen: Length 9.9-11.8 mm ( $\mathrm{N}=7)$, width 4.1-4.2 mm ( $\mathrm{N}=2)$. Cylindrical, dark brown, probably black in life. Tergites and sternites with rather long, brown pile.

Terminalia: Cerci rather small, apically rounded. 


\section{Plecia rhenana Heyden \& Heyden, 1865 (Fig. 49, 160-161)}

Plecia rhenana Heyden \& Heyden, 1865:

Plecia robusta Brongniart, 1879 - presumably an unpublished cabinet name in collection of EM

Material examined: Holotype of Plecia rhenana (female) NHMUK In 38798.

Additional material: 4 females, Coll. Coquand, EM: EM 70007, 70016, 70018, 70206 (A 10.14).

\section{Localities: Aix-en-Provence (Rott)}

The species was described from Rott, Germany. It is seemingly not present in the material from Aix in MNHN, but there are four females in Coll. Coquand, EM (temporarily stored at FSL) which are probably conspecific with the holotype.

\section{Diagnosis:}

\section{Redescription:}

Male unknown.

Female: Total length $10.5-12.3 \mathrm{~mm}(\mathrm{~N}=4)$.

Head (fig. 161): Length 0.91-1.01 mm (N=3), width 0.97-1.10 mm (N=3). Flagellum length $0.68 \mathrm{~mm}$ $(\mathrm{N}=1)$.

Thorax: Length 2.8-3.0 mm (N=4), width 2.3-2.5 mm (N=3).

Wing (fig. 160): Length 8.7-9.5 mm (N=4), width 3.0-3.6 mm ( $=4)$, length/width $=2.63-2.93$ $(\mathrm{N}=4)$. Vein measurements in Table 3.

Legs: Length of fore femur $2.5 \mathrm{~mm}(\mathrm{~N}=1)$, fore tibia $1.7-2.5 \mathrm{~mm}(\mathrm{~N}=3)$.

Abdomen: Length 7.6-9.4 mm (N=4), width 2.5-3.0 mm (N=3).

\section{Genus Bibio Geoffroy, 1762}

The genus is usually easily recognized by the wing venation, the shape of the anterior tibiae as well as the general habitus. Surprisingly, we still found that a number of described species belonging to this genus had been placed in other genera, and some species placed in Bibio belonged to other genera. Bibio species are present from most localities except Chadrat and Barbeiras. In some (e.g., Aix-enProvence and Monteils) they predominate while in others (e.g. Céreste and Bois d'Asson) they are relatively infrequent.

Bibio aquaesextiae sp.n. (figs. 50, 162-164)

Material examined: Holotype (male) MNHN Aix 152. 
Paratypes (males) MNHN Aix 610, 1294, 1436, 1452, (females) MNHN Aix 309, 416, 421, 532 , $1010,1014$.

Localities: Aix-en-Provence

Diagnosis: A large (9-13.5 mm) Bibio with relatively slender body. Antennal flagellum 8-segmented. Wing hyaline with crossvein R-M nearly as long as basal section of Radial sector. Male hind tibia strongly clavate. Epandrium bilobate, gonostylus rather small.

Etymology: The species is named after Aquae Sextiae, the Roman colony which later developed into Aix-en-Provence.

\section{Description:}

Male: Total length $8.5-12.0 \mathrm{~mm}(\mathrm{~N}=5)$. General body shape slender.

Head (fig. 163): Length 1.1-1.5 mm, width 1.2-1.7 mm (N=5). Complex eye large, intraocular pile not conspicuous. Ocelli rather small and widely spaced. Antenna slender, flagellum 8-segmented, $0.56-0.67 \mathrm{~mm}$ long $(\mathrm{N}=3)$.

Thorax: Length 2.3-2.6 mm, width 2.0-2.3 mm (N=5). Black. Haltere pale.

Wing (fig. 162): Length 6.4-8.9 mm, width 2.8-3.5 mm ( $\mathrm{N}=5)$, length/width $=2.18-2.49$ (N=5). Apparently hyaline, veins fine and concolourous with membrane. Pterostigma rather indistinctive. Crossvein R-M nearly as long as basal section of Rs. $\mathrm{CuA}_{1}$ and $\mathrm{CuA}_{2}$ apically curved. Wing vein measurements in Table 4.

Legs: Probably pale coloured, relatively long. Fore tibia relatively slender. Hind tibia strongly clavate. Fore femur length $1.5-2.2 \mathrm{~mm}$, width $0.55-0.66 \mathrm{~mm}(\mathrm{~N}=3)$, fore tibia length $1.6-2.0 \mathrm{~mm}(\mathrm{~N}=3)$, width $0.32 \mathrm{~mm}(\mathrm{~N}=1)$, hind femur 2.1-3.2 mm, width 0.43-0.55 $\mathrm{mm}(\mathrm{N}=4)$, hind tibia 2.3-3.4 mm, width $0.53-0.62 \mathrm{~mm}(\mathrm{~N}=5)$.

Abdomen: Length 5.1-7.4 mm, width 1.4-1.9 mm ( $\mathrm{N}=5)$. Long and slender, tapering apicad, brown.

Terminalia (fig. 164): Epandrium bilobate with rounded lobes, indentation between the lobes wide and U-shaped, laterally with a small indentation. Gonostyli relatively short. Ventrally with wide, rounded indentation between gonocoxites. Hypopygium width $0.8-1.1 \mathrm{~mm}(\mathrm{~N}=5)$, gonostylus length $0.40-0.47$ $\mathrm{mm}(\mathrm{N}=4)$.

Female: Total length $10-13.5 \mathrm{~mm}(\mathrm{~N}=5)$.

Head: Length 1.17-1.26 mm ( $=3)$, width 0.93-1.31 mm ( $=4)$. Rounded, complex eye relatively large, not protruding. Antennal flagellum 8-segmented.

Thorax: Length 2.3-2.5 mm ( $=5)$, width 2.1-2.5 mm (N=4). Brown, haltere pale.

Wing: Length 7.7-9.8 mm (N=5), width 2.9-4.0 mm (N=5). Presumably hyaline, veins fine and concolourous with membrane. Pterostigma light brown, not very conspicuous. Venation as in male. Wing vein measurements in Table 4.

Legs: Femorae brown, tibia and tarsi apparently lighter. Hind femur thick, clavate, hind tibia slender. Fore femur length $1.6-2.4 \mathrm{~mm}(\mathrm{~N}=4)$, width $0.73-0.81 \mathrm{~mm}(\mathrm{~N}=2)$, fore tibia length $1.9-2.2 \mathrm{~mm}$ 
$(\mathrm{N}=2)$, width $0.46 \mathrm{~mm}(\mathrm{~N}=1)$, hind femur length $2.2-3.5 \mathrm{~mm}(\mathrm{~N}=4)$, width $0.59-0.69 \mathrm{~mm}(\mathrm{~N}=4)$, hind tibia length $2.5-3.6 \mathrm{~mm}(\mathrm{~N}=4)$, width $0.41-0.58 \mathrm{~mm}(\mathrm{~N}=4)$.

Abdomen: Length 6.4-9.4 mm (N=5), width 2.1-3.2 mm (N=4). Relatively slender, cylindrical.

Colour probably light brown.

Terminalia: Three spermathecae present as usual. Cerci small, rounded.

Discussion: None of the specimens at hand show good colour preservation and it is possible that colours have been considerably darker in life.

\section{Bibio baumbergeri Théobald, 1937 (Figs. 51-52, 165-171)}

Bibio baumbergeri Théobald, 1937: 223.

Bibio parenti Théobald, 1937: 224. Syn.n.

Bibio bayleyi Brongniart, 1879, presumably an unpublished cabinet name in collection of EM

Material examined: Holotype (female) of Bibio baumbergeri NMB F456. Paratype (female) of Bibio baumbergeri NMB. Holotype (female) of Bibio parenti NMB F1372. Female (EM 70001, stored at FSL), labeled "Bibio Bayleyi".

Additional material - 4 males NMB F1377/4, F1377/6, F2183, F2258; 7 females: NMB F1376/1-2, F1377/2-3, F1377/5, F454, F 2330. 8 females, Aix-en-Provence, Coll. Coquand, EM 70207 (A 10.3), 70208 (A 10.6), 70209 (A 10.44), 70210 (301 ג), 70211-70214.

Localities: Kleinkembs, Aix-en-Provence.

Diagnosis: A medium-sized to large $(8.3-13 \mathrm{~mm})$, robust Bibio. Antennal flagellum 7-segmented. Wing hyaline to yellowish fumose with concolourous veins, pterostigma indistinctive, crossvein R-M about one third as long as basal Rs. Legs yellowish-brown in female, black in male, protibial spur about half as long as anterior spine.

\section{Redescription:}

Male: Total length $8.3-10.3 \mathrm{~mm}(\mathrm{~N}=4)$.

Head: Black. Ocellar triangle low and inconspicuous.

Thorax: Length 2.7-2.9 mm ( $\mathrm{N}=3)$, width $2.3 \mathrm{~mm}(\mathrm{~N}=1)$. Black, haltere pale yellowish.

Wing (fig. 165): Length $6.8 \mathrm{~mm}$, width $2.8 \mathrm{~mm}(\mathrm{~N}=1)$. Hyaline, R-veins brown, fine, posterior veins colourless. Basal section of $\mathrm{R}_{4+5}$ about three times as long as R-M. Wing vein measurements in Table 4.

Legs: Black. Fore femur 2.0-2.3 mm long, $0.7 \mathrm{~mm}$ wide ( $\mathrm{N}=2)$. Hind femur stout, clavate, hind tibia slender. Length of hind femur 2.4-3.5 mm, width $0.5-0.6 \mathrm{~mm}(\mathrm{~N}=3)$, length of hind tibia $2.2-2.9 \mathrm{~mm}$ $(\mathrm{N}=2)$. 
Abdomen: Black.

Terminalia (fig. 171): Not well preserved in any of the specimens at hand. Epandrium bilobate with wide, U-shaped cleft between lobes.

Female: Total length 9.3-13.0 mm (N=9).

Head (fig. 167): Length 1.2-1.6 mm (N=4), width 1.1-1.2 mm ( $\mathrm{N}=2)$, longer than broad, nearly rectangular. Complex eye small and prominent. Ocellar tubercle rather small. Antenna rather small.

Thorax: Length 2.7-4.0 mm (N=8), width $2.7 \mathrm{~mm}(\mathrm{~N}=1)$. Black.

Wing (fig. 166): Length 8.0-10.7 mm (N=8), width 3.1-3.7 mm (N=6). Yellowish fumose, R-veins yellowish, posterior veins colourless. Subcosta rather short, apically indistinctive. Costa extends to apex of $\mathrm{R}_{4+5}$.Pterostigma indistinctive. Basal radial sector about three times as long as R-M. Wing vein measurements in Table 4.

Legs (figs. 168-169): Yellowish brown to dark brown. Fore femur length 1.9-2.6 mm (N=6), width $0.71-0.91 \mathrm{~mm}(\mathrm{~N}=6)$. Fore tibia length $2.1-2.2 \mathrm{~mm}(\mathrm{~N}=2)$, width $0.41-0.56 \mathrm{~mm}(\mathrm{~N}=2)$, with spine approximately twice as long as spur. Hind femur length $2.4-4.5 \mathrm{~mm}(\mathrm{~N}=10)$, width $0.46-0.71 \mathrm{~mm}$ $(\mathrm{N}=10)$, clavate, hind tibia length $2.2-4.2 \mathrm{~mm}(\mathrm{~N}=7)$, slender, tarsi slender.

Abdomen: Brown, cylindrical.

Terminalia (fig. 170): Cerci short, curved and pointed.

\section{Bibio celasensis Théobald, 1937 (Figs. 53-55, 172-177)}

Bibio celasensis Théobald, 1937: 139-140.

Bibio conformans Théobald, 1937: 142. Syn.n.

Bibio obtusa Theobald 1937:140 and Bibio tenuiapicalis Theobald, 1937: 140 are also probably synonyms for this species, but we have not succeeded in finding the types of these species.

Material examined: Syntypes of Bibio celasensis - more than 100 specimens on a plate $12 X 14 \mathrm{~cm}$ (MHNM). Most of them poorly preserved. Bibio conformans - specimen (female) depicted by Théobald (1937, Pl. XXI, Fig. 12) FSL 392012 - A19.

Additional material: Males: MNHN Monteils 1 (counterpart Monteils 61), 54, 59, 79, 99 (counterpart Monteils 106), 114, Camoins 29. Females: MNHN Monteils 8, 15, 17, 19, 22, 23, 27, 28, 42, 48, 49, $52,60,62,66,83,93,101,107,110,121,127,130,132,137,141,160,167$, Célas 1, 2 females of uncertain origin.

Localities: Célas, Camoins, Monteils

Diagnosis: A medium-sized (6-9 mm) Bibio. Antenna short and robust, flagellum 7-segmented. Legs with femorae black, stout and clavate. Protibial spur about one third as long as anterior spine. Wing hyaline with dark R-veins, posterior veins colourless, basal part of $R_{s}$ two to three times as long as RM. 


\section{Redescription:}

Male (fig. 173): Total length 6.0-7.5 mm (N=7).

Head: Length 1.04-1.17 mm (N=5), width $1.17 \mathrm{~mm}(\mathrm{~N}=1)$. Antenna short and robust, flagellum probably 7-segmented.

Thorax: Length 2.1-2.5 mm (N=5). Dark brown, presumably black in life. Haltere pale.

Wing (fig. 172): Length 4.1-5.2 mm ( $\mathrm{N}=3)$, width $1.9 \mathrm{~mm}(\mathrm{~N}=1)$. R-veins brown, posterior veins colourless. Costa extends slightly beyond $\mathrm{R}_{4+5}$. Basal $\mathrm{R}_{\mathrm{s}}$ approximately as long as R-M. Pterostigma rather small, brown, distinctive.

Legs: Hind femur clavate, hind tibia apically truncate, rather slim. Legs uniformly dark brown. Fore femur 1.3-1.7 mm long $(\mathrm{N}=4), 0.44-0.58 \mathrm{~mm}$ wide $(\mathrm{N}=4)$, fore tibia $1.4 \mathrm{~mm}$ long $(\mathrm{N}=1)$, hind femur 1.9-2.2 mm long $(\mathrm{N}=5), 0.42-0.55 \mathrm{~mm}$ wide $(\mathrm{N}=5)$, hind tibia 1.9-2.0 $\mathrm{mm}$ long $(\mathrm{N}=3), 0.35-0.38 \mathrm{~mm}$ wide $(\mathrm{N}=3)$, hind first tarsomere $0.67 \mathrm{~mm}$ long $(\mathrm{N}=1)$.

Abdomen: Length 3.6-5.4 mm (N=5). Relatively slender.

Teminalia (fig. 176): Hypopygium width $0.80-0.84 \mathrm{~mm}(\mathrm{~N}=2)$. Gonostylus rather short, curved and digitiform as usual for genus.

Female (fig. 174): Total length 7.5-11.0 mm (N=28).

Head (fig. 175) Length 1.04-1.38 mm ( $\mathrm{N}=24)$, width 0.81-1.21 mm ( $\mathrm{N}=4)$. Antenna rather short, flagellum 7-segmented, 0.41-0.58 mm long $(\mathrm{N}=5)$. Ocellar tubercle low, not prominent. Complex eye medium-sized.

Thorax: Length 2.1-2.9 mm (N=28). Dark brown to black, probably black in life. Haltere pale brown.

Legs: Femorae dark brown, probably black in life, tibiae and tarsi brown. Femorae stout and spindleshaped, hind tibia rather slender, tarsi slender. Protibia stout, spur short, approximately $1 / 3$ the length of spine. Fore femur 1.5-1.8 mm long $(\mathrm{N}=16), 0.54-0.86 \mathrm{~mm}$ wide $(\mathrm{N}=16)$, fore tibia 1.6-1.8 $\mathrm{mm}$ long $(\mathrm{N}=4)$, hind femur 1.9-2.7 mm long $(\mathrm{N}=24), 0.47-0.65 \mathrm{~mm}$ wide $(\mathrm{N}=25)$, hind tibia 1.9-2.6 $\mathrm{mm}$ long $(\mathrm{N}=24), 0.25-0.48 \mathrm{~mm}$ wide $(\mathrm{N}=22)$, hind first tarsomere $0.59-0.73 \mathrm{~mm}$ long $(\mathrm{N}=11)$. The great variability in leg width is probably due to variations in the angle in which the legs are preserved, this is very difficult to judge in flattened fossils.

Wing: Length 5.4-7.3 mm ( $\mathrm{N}=15)$, width 1.9-2.8 mm ( $\mathrm{N}=10)$, hyaline, $\mathrm{R}$-veins brown, posterior veins pale brown to colourless. Pterostigma rather small, brown. Basal part of $R_{\mathrm{s}}$ approximately as long as R-M.

Abdomen: Length 4.1-7.8 mm $(\mathrm{N}=26)$, cylindrical, rather stout, brown.

Terminalia (fig. 177): Relatively small and inconspicuous, cerci rounded.

\section{Bibio curtisii Heer, 1856 (Figs. 56-61, 178-183)}

Bibio curtisii Heer 1856: 34-35.

Bibio martinsi Heer in Heer \& Gaudin, 1861: 153 (nomen nudum)

Bibio ungeri marginatus Oustalet, 1870: 125. Syn.n.

Bibio romani Théobald, 1937: 336 Syn.n.

Bibio serresi Théobald, 1937: 336 Syn.n. 
Material examined: Lectotype (herein designated) male NHMUK In 39833. This specimen was illustrated by Lyell \& Murchison (1829) and later by Heer (1856, Fig. 14). Specimen NHMUK In 39855 is the counterpart of the lectotype. Paralectotypes (males, herein designated) NHMUK In 39825/39845 (part and counterpart), ETHZ S.N. 158 (part and counterpart). Holotype (female) of Bibio romani (FSL 391915). Paratype (male) of Bibio serresi (FSL 391905). Bibio ungeri v. marginatus (depicted specimen, male, Théobald 1937) FSL 391918, A 26, (female) FSL 391919, A38, (female) FSL 391920, A 87, (female) EEFN Coll. Fliche F 201.

Other material: 1 male (ETHZ S.N. 131), 1 male (FSL 391904), 3 males (MNHN Aix 42, Aix 54, Aix 584), one pair of wings, presumably male (ETHZ S.N. 137), 2 females (FSL 391908, 391909), 4 females (ETHZ S.N. 131), 2 females (MNHN Aix 873, Aix 950), 1 female (MNHN B 24431).

While Bibio ungeri marginatus is synonymous with this species, Bibio ungeri s.str. is obviously another species entirely. A specimen in the Museum of Natural History, Vienna is a Penthetria species, while Heer's figure (Heer 1849: Plate XVI: 8) is of a specimen in Landesmuseum Joanneum, Graz which is obviously not a bibionid. This large and slender specimen, which is rather poorly preserved with head and legs missing, is apparently a brachyceran, and possibly belongs in Xylophagidae or Asilidae. The wing venation is hard to see but apparently there is a pointed anal cell and a closed discal cell in the wing.

Localities: Aix-en-Provence.

Diagnosis: A medium-sized-large Bibio (9.5-12 mm). Head normal-sized for genus, antennal flagellum seven-segmented. Male complex eye with conspicuous, long, dark pile. Wing hyaline with dark brown veins, humeral crossvein present between Sc and Costa in basal third, pterostigma black, conspicuous. Crossvein R-M short but distinctive. Veins $\mathrm{CuA}_{1}$ and $\mathrm{CuA}_{2}$ apically distinctively curved. Legs all black. The species is habitually rather similar to Bibio crassinervis Skartveit \& Pika, 2014 from the Miocene site of Öhningen, but differs from that in having an obvious humeral crossvein, which is absent in B. crassinervis, as well as having the wing veins not quite as strong and prominent.

\section{Redescription:}

Male: Total length $8.5-11.6 \mathrm{~mm}(\mathrm{~N}=30)$

Head (fig. 180): Length 1.43-1.87 mm (N=9), width 1.64-1.97 mm ( $=7)$. Complex eye with dense and rather long, brownish pile. Antennal flagellum probably 7-segmented. Palp robust.

Thorax: Length 2.9-3.8 mm ( $=10)$, width 2.4-2.8 mm ( $\mathrm{N}=3)$. Black, dorsally with long, dark pile. Haltere dark brown.

Wing (fig. 178): Length 6.3-7.9 mm ( $\mathrm{N}=11)$, width 2.6-3.5 mm ( $\mathrm{N}=11)$, length/width $=2.38-2.65$ $(\mathrm{N}=11)$. Hyaline to light brownish, veins blackish, prominent throughout. Humeral vein prominent. Pterostigma brown, oval, prominent. Costa terminates just after apex of $\mathrm{R}_{4+5}$. Basal part of Radial sector about three times as long as R-M. Crossvein $\mathrm{M}-\mathrm{Cu}$ joins $\mathrm{M}_{2}$ slightly beyond fork of $\mathrm{M}$. $\mathrm{CuA}_{1}$ apically curved backwards. Wing vein measurements in Table 4. 
Legs (figs. 181-182): Dark brown, presumably black in life. Protibia with spine about three times as long as spur. Hind femur moderately stout and clavate, hind tibia evenly expanding towards apex, not conspicuously clavate, with short, robust spur. Hind tarsus slender. Fore femur 1.7-2.3 mm long $(\mathrm{N}=3), 0.55-0.72 \mathrm{~mm}$ wide $(\mathrm{N}=3)$, fore tibia $1.4-1.7 \mathrm{~mm}$ long $(\mathrm{N}=2)$, hind femur 2.4-3.6 $\mathrm{mm}$ long $(\mathrm{N}=7), 0.47-0.66 \mathrm{~mm}$ wide $(\mathrm{N}=7)$, hind tibia $2.7-3.7 \mathrm{~mm}$ long $(\mathrm{N}=5), 0.50-0.58 \mathrm{~mm}$ wide $(\mathrm{N}=5)$, hind first tarsomere $0.9-1.0 \mathrm{~mm}(\mathrm{~N}=2)$.

Abdomen: Length 5.6-6.9 mm $(\mathrm{N}=10)$, width 1.6-2.0 mm $(\mathrm{N}=5)$. Dark brown, presumably black in life.

Terminalia (fig. 183): Epandrium 0.97-1.16 mm wide ( $\mathrm{N}=5$ ), with wide, shallow, $\mathrm{U}$-shaped emargination, lateral lobes short and blunt. Gonostylus robust, of the typical shape for the genus.

Female: Total length 10.0-12.0 mm (N=6).

Head: Length 1.08-1.22 mm ( $\mathrm{N}=3)$, width $1.05-1.30 \mathrm{~mm}(\mathrm{~N}=2)$. Antennal flagellum 7-segmented. Thorax: Length 2.9-3.8 mm (N=6), width 2.6-2.7 mm ( $=2)$. Dark brown, presumably black in life. Haltere brown.

Wing (fig. 179): Length 7.8-9.9 mm (N=6), width 3.2-3.5 mm ( $\mathrm{N}=5)$, length/width $=2.44-2.82$ $(\mathrm{N}=5)$. Light brownish hyaline, veins strong and blackish throughout, pterostigma blackish. Venation as in male. Wing vein measurements in Table 4.

Legs: Dark brown, presumably black in life. Hind femur robust, clavate, hind tibia slender. Fore femur length 1.8-2.3 mm $(\mathrm{N}=5)$, width $0.59-0.77 \mathrm{~mm}(\mathrm{~N}=3)$, fore tibia length $1.8-2.3 \mathrm{~mm}(\mathrm{~N}=4)$, hind femur length 2.4-3.2 $\mathrm{mm}(\mathrm{N}=4)$, width $0.54-0.64 \mathrm{~mm}(\mathrm{~N}=4)$, hind tibia length $2.5-3.2 \mathrm{~mm}(\mathrm{~N}=5)$, width $0.32-0.43 \mathrm{~mm}(\mathrm{~N}=3)$.

Abdomen: Length 6.7-8.4 mm ( $\mathrm{N}=6)$, width 2.3-2.9 mm $(\mathrm{N}=3)$. Dark brown, presumably black in life. Parallel-sided, the two terminal tergites narrower. Cerci prominent, pointed.

\section{Bibio dislocatus Théobald, 1937 (Figs. 62-64, 184-187)}

Bibio dislocatus Théobald, 1937: 224-225.

Bibio consolida Théobald, 1937: 225. Syn.n.

Plecia macroventris Théobald, 1937: 229. Syn.n.

Material examined: Holotype (female) of Bibio dislocatus NMB F1378. Holotype (female) of Bibio consolida NMB F1379. Holotype (female) of Plecia macroventris NMB F1383.

Additional material (3 females) NMB F1385/1-2, F1386/1-2.

Localities: Kleinkembs.

Diagnosis: A medium-sized (8.0-9.0 mm), robust Bibio. Antennal flagellum possibly 7-segmented. Fore tibia with spur about half as long as anterior spine. Wing hyaline, veins pale, pterostigma indistinct, crossvein R-M about as long as basal section of Rs.

\section{Redescription:}


Male unknown.

Female (fig. 185): Total length 8.0-9.0 mm (N=3), robust. Entirely black.

Head (fig. 186): Length 0.95-1.03 mm ( $\mathrm{N}=4)$, width $1.06 \mathrm{~mm}(\mathrm{~N}=1)$. Black, widest behind middle, considerably narrowing forwards. Ocellar tubercle indistinct. Antenna rather small, flagellum possibly 7-segmented, $0.63 \mathrm{~mm}$ long $(\mathrm{N}=1)$.

Thorax: Length 2.4-2.6 mm ( $\mathrm{N}=4)$. Black, relatively flat dorsally.

Wing (fig. 184): Length 5.9-7.5 mm ( $\mathrm{N}=2)$. Hyaline, R-veins light brown, posterior veins colourless. Pterostigma pale brown, scarcely visible. Costa extends slightly beyond apex of $\mathrm{R}_{4+5}$. Basal section of $\mathrm{R}_{\mathrm{s}}$ subequal to crossvein $\mathrm{R}-\mathrm{M}$.

Legs: Brown to black, most likely all black in life. Fore tibia with spur a little over half as long as spine. Femorae strongly clavate, hind tibia and all tarsi slender. Fore femur 1.6-1.7 mm long $(\mathrm{N}=3)$, 0.58-0.71 mm wide $(\mathrm{N}=3)$, fore tibia 1.6-1.7 $\mathrm{mm}$ long $(\mathrm{N}=3)$, hind femur 2.1-2.3 $\mathrm{mm}$ long $(\mathrm{N}=4)$, $0.32-0.38 \mathrm{~mm}$ wide $(\mathrm{N}=4)$, hind first tarsomere $0.66-0.77 \mathrm{~mm}$ long $(\mathrm{N}=4), 0.25 \mathrm{~mm}$ wide $(\mathrm{N}=1)$.

Abdomen: Length 5.6-6.2 mm (N=3). Brown, most likely black in life, cylindrical, rather stout. Terminalia (fig. 187): Cerci small and rounded.

\section{Bibio fusiformis Heer, 1849}

This species was described from the Miocene of Öhningen, Southern Germany and redescribed by Skartveit \& Pika (2014). The species was recorded with some doubt from Aix-en-Provence by Heer (1856: 33). We have not seen any specimens of this species from French localities and judging from Heer's (1856) brief description of the specimen it is almost certainly not identifiable at the species level. Its presence in French Oligocene sites is thus unproven and we regard it as unlikely. A specimen identified as Bibio cf. fusiformis and illustrated by Théobald (1937, Pl. XXI, Fig. 10) in FSL (FSL 391917) fits the specimens from Öhningen reasonably well but is not well enough preserved for a certain identification. Another specimen (BMNH In 39823) in BMNH is also in too poor condition to identify the species with any degree of certainty. Furthermore, there are four female specimens in MNHM (labeled with the numbers 209-212) identified as Bibio fusiformis. These share with the type specimens the conspicuously slender, cylindrical abdomen but are otherwise devoid of systematically useful characters.

\section{Bibio major (Oustalet, 1870) comb.n. (Figs. 65-67, 188-197)}

Plecia major Oustalet, 1870: 114

Bibio inflexus Théobald, 1937: 404.

Material examined: Plecia major syntype (male) MNHN R 06693. Bibio inflexus holotype (female) EEFN Coll. Fliche F 273-274.

Additional material: 41 males: UPMC Céreste 75; (all following RGL, currently stored in MNHN) Céreste 34, 74, 87, 90, 223, 269, 316, 338, 353, 554, 644, 649, 660, 664, 665, 666, 718, 896, 991, 992, 993, 995, 1000, 1019, 1026, 1029, 1030, 1116, 1144, 1166, 1419, 1742, P91, P188, P254, P353, P452, P516, P526, P539. 36 females: Céreste 54, 68, 76, 275, 568, 639, 641, 653, 654, 655, 656, 667, 1011, 1018, 1020, 1021, 1027, 1028, 1099, 1100, 1111, 1134, 1360, 1574, 1655, 1672, 1685, 1697, P316, P358, P418, P461, P519, P534, P543, P551.

Localities: Corent, Céreste 
Diagnosis: a medium-sized to large $(8.5-13 \mathrm{~mm})$ Bibio species. Head conspicuously small, width in male about half of thorax width, flagellum slender and 10-segmented. Wing hyaline, vein Rs dips toward M so that crossvein R-M is obliterated, but the two veins are not fused for any distance like in the genus Bibiodes. Pterostigma small, dark and distinctive. Male legs entirely dark, fore tibia with spur approximately half as long as anterior spine, hind femur thick and spindle-shaped, hind tibia rather slender, not clavate, apically truncate. Male epandrium small and not bilobate. Female body dark, legs mostly pale-coloured.

\section{Redescription:}

Male (figs. 190-191): Total length 8.5-11.2 mm (N=31). Entirely black, body pile not conspicuous. Head (fig. 193): Length $0.87-1.35 \mathrm{~mm}(\mathrm{~N}=10)$, width $0.98-1.35 \mathrm{~mm}(\mathrm{~N}=9)$. Conspicously small, width approximately half of thorax width. Flagellum 10 -segmented, length $0.49-0.68 \mathrm{~mm}(\mathrm{~N}=5)$.

Thorax: Length 2.4-3.4 mm ( $\mathrm{N}=20)$, width 2.0-2.8 mm ( $\mathrm{N}=5)$. Black. Haltere pale brownish.

Wing (fig. 188): length 6.2-8.0 mm ( $\mathrm{N}=9)$, width 2.7-3.4 mm ( $=5)$, length/width $=2.12-2.60(\mathrm{~N}=5)$, hyaline, R-veins brown, posterior veins colourless. Pterostigma small, dark and distinctive. Wing vein measurements in Table 4.

Legs (figs. 194-195): Black. Mid- and hind femorae moderately clavate, hind tibia rather slender, straight-sided, apically truncate. Hind first tarsomere slender to moderately robust. Fore femur length 1.4-2.0 mm (N=5), width 0.53-0.65 mm ( $\mathrm{N}=5)$, fore tibia length $1.5-1.6 \mathrm{~mm}(\mathrm{~N}=5)$, fore first tarsomere length $0.9-1.4 \mathrm{~mm}(\mathrm{~N}=4)$, hind femur length $2.3-3.5 \mathrm{~mm}(\mathrm{~N}=20)$, width $0.51-0.65 \mathrm{~mm}$ $(\mathrm{N}=13)$, hind tibia length $2.3-3.1 \mathrm{~mm}(\mathrm{~N}=22)$, width $0.34-0.55 \mathrm{~mm}(\mathrm{~N}=14)$, hind first tarsomere length $0.81-0.93 \mathrm{~mm}(\mathrm{~N}=6)$.

Abdomen: Length 5.2-7.1 mm ( $\mathrm{N}=10)$, width 1.2-1.6 mm (N=8). Black.

Terminalia (fig. 196): Hypopygium width 0.85-1.06 mm ( $\mathrm{N}=8)$. Epandrium rather small, posteriorly concave but not bilobate, posterior corners slightly protruding. Gonostyli robust, quite long, of the typical shape for genus.

Female (fig. 192): Total length 10.0-13.0 mm (N=12).

Head: Length 1.03-1.30 mm ( $=7)$, width 1.13-1.43 mm ( $\mathrm{N}=7)$, quite short and broad. Flagellum 10segmented, length $0.67-0.84 \mathrm{~mm}(\mathrm{~N}=5)$.

Thorax: Length 3.2-4.5 mm (N=11), width 2.4-3.2 $\mathrm{mm}(\mathrm{N}=5)$.

Wing (fig. 189): Length 7.9-10.5 mm (N=6), width 2.9-3.7 mm (N=4), length/width $=2.42-2.71$

$(\mathrm{N}=4)$. Hyaline, venation as in male. Pterostigma small and dark, distinctive. Wing vein measurements in Table 4.

Legs: Legs mostly pale. Fore femur 2.1-2.4 mm long ( $\mathrm{N}=2), 0.76-0.79 \mathrm{~mm}$ wide $(\mathrm{N}=2)$, fore tibia 2.1-2.3 mm long ( $\mathrm{N}=3)$, fore first tarsomere $1.5 \mathrm{~mm}(\mathrm{~N}=1)$, hind femur 2.9-3.6 mm long $(\mathrm{N}=4), 0.61-$ $0.70 \mathrm{~mm}$ wide $(\mathrm{N}=5)$, hind tibia 3.1-3.6 $\mathrm{mm}$ long $(\mathrm{N}=6), 0.40-0.49 \mathrm{~mm}$ wide $(\mathrm{N}=4)$, hind first tarsomere $1.0-1.2 \mathrm{~mm}(\mathrm{~N}=3)$.

Abdomen: Length 6.0-8.6 mm ( $\mathrm{N}=11)$, width 2.1-3.1 mm ( $\mathrm{N}=7)$.

Terminalia (fig. 197): Last sternite with a narrow and shallow indetation. 
The wing venation of this species approaches the typical venation of the genus Bibiodes Coquillett, which, though only found in the Nearctic region in the recent fauna, is known from several European fossils (Skartveit 2009). Unfortunately, in most of the specimens from Céreste and Aix-en-Provence the wing venation is not visible. But the male genitalia are typical of the genus Bibio and $\mathrm{R}_{\mathrm{s}}$ and $\mathrm{M}$ just meet without really merging, hence it is classified as a Bibio species.

\section{Bibio moestus Heer, 1849}

This species was described from Öhningen, Southern Germany and redescribed by Skartveit \& Pika (2014). It was recorded from Aix-en-Provence by Heer (1856: 33-34), one specimen is in ETHZ (Skartveit \& Pika 2014). Since Heer did not illustrate the specimen from Aix-en-Provence it is uncertain if the specimen in ETHZ is the one recorded by Heer, though the specimen is labelled in Heer's handwriting. The Aix specimen in ETHZ labeled as B. moestus is most likely not conspecific with the specimens from Öhningen which form the type series (Skartveit \& Pika 2014), but is probably a female of Bibio curtisii. The presence of B. moestus in French Oligocene sites is thus unproven, and we regard it as unlikely.

\section{Bibio spectabilis Théobald, 1937 (figs. 68, 198-206)}

Bibio spectabilis Théobald, 1937: 333.

Material examined: Holotype (female) of Bibio spectabilis FSL 391911. Paratype (female) of Bibio spectabilis FSL 391912.

Additional material: Male (tentatively associated) EM 70215 (1.2.2) (stored in FSL). Males (MNHN Aix 52, 1377). Females (MNHN Aix 6, 10, 11, 107, 161, 309, 845, 1090, 1299, 1307, 1446, 1447, $\mathrm{P} 25)$.

Localities: Aix-en-Provence

Diagnosis: A medium-sized to large $(8.5-12 \mathrm{~mm})$, robust Bibio species. The wing has a short but distinctive R-M crossvein, about one fifth as long as basal part of $\mathrm{R}_{\mathrm{s}}$, and $\mathrm{CuA}_{1}$ and $\mathrm{CuA}_{2}$ are apically straight or nearly so. Antenna robust with 8-segmented flagellum. Male epandrium not bilobate, apically straight with slightly protruding posterior corners.

\section{Redescription:}

Male (tentatively associated, fig. 200): Total length 9.0-10.0 mm ( $\mathrm{N}=3)$.

Head (fig. 201): Length 1.3-1.6 mm (N=2), width $1.5 \mathrm{~mm}(\mathrm{~N}=1)$. Dark brown, presumably black in life. Antennal flagellum 8-segmented. Palp relatively short and robust. Ocellar triangle large and prominent.

Thorax: Length 2.8-3.1 mm (N=2), width $2.3 \mathrm{~mm}(\mathrm{~N}=1)$. Dark brown, presumably black in life. Haltere pale.

Wing (fig. 199): Length 5.8-6.9 mm (N=3), width 2.5-2.8 $\mathrm{mm}(\mathrm{N}=3)$, length/width $=2.31-2.73$ $(\mathrm{N}=3)$. Hyaline? Membrane pigmentation not well preserved, veins distinctive throughout. $\mathrm{R}_{4+5}$ arched forwards, running more or less parallel to Costa in distal half. Crossvein R-M short but distinctive. $\mathrm{CuA}$-veins apically straight or nearly so. Wing vein measurements in Table 4. 
Legs (fig. 202): Fore tibia with spur a little less than half as long as anterior spine. Hind femur stout, clavate. Hind tibia rather short and robust with long, curved, apically blunt spur. Hind tarsus robust, first tarsomere approximately three times as long as wide. Hind femur length $2.5-2.7 \mathrm{~mm}(\mathrm{~N}=3)$, width $0.52-0.60 \mathrm{~mm}(\mathrm{~N}=3)$, hind tibia length $2.3-2.9 \mathrm{~mm}(\mathrm{~N}=3)$, width $0.40-0.44 \mathrm{~mm}(\mathrm{~N}=3)$.

Abdomen: Length 5.8-6.2 mm ( $\mathrm{N}=3)$, width $1.3 \mathrm{~mm}(\mathrm{~N}=1)$. Dark brown, presumably black in life, conical.

Terminalia (fig. 203): Hypopygium width $0.92-1.10 \mathrm{~mm}(\mathrm{~N}=2)$. Epandrium not bilobate, posterior margin nearly straight with rounded, slightly protruding posterior corners. Gonostylus large and robust.

Female: Total length 9.3-12.0 mm (N=13)

Head (fig. 204): Length 1.19-1.39 mm (N=6), width 0.93-1.32 mm (N=6). Black. Antennal flagellum 0.55-0.62 mm long ( $\mathrm{N}=2), 8$-segmented. Ocellar tubercle small and low. Palp short and robust.

Complex eye rather small, about one third length of head.

Thorax: Length 2.8-3.8 mm ( $\mathrm{N}=13)$, width 2.1-3.1 mm ( $\mathrm{N}=5)$. Haltere dark brown.

Wing (fig. 198): Length 6.7-10.6 mm ( $\mathrm{N}=14)$, width 3.0-4.3 mm ( $\mathrm{N}=14)$, length/width $=2.08-2.62$ $(\mathrm{N}=14)$. Grayish hyaline, pterostigma oval, dark brown, wing mesally with a chocolate-brown band stretching from the origin of $\mathrm{R}_{\mathrm{s}}$ distad to pterostigma, posteriorly to $\mathrm{CuA}_{1}$ Crossvein $\mathrm{R}-\mathrm{M}$ short but distinctive, about one fifth as long as basal section of $\mathrm{R}_{\mathrm{s}}$. The CuA-veins apically straight or nearly so. Wing vein measurements in Table 4.

Legs (fig. 205): femorae and tibiae brownish yellow, tibiae apically darker. Fore femur relatively slender, about three times as long as wide in lateral view. Fore tibia with spur about one third the length of anterior spine. All tarsi slender, tarsomeres brownish yellow, apically darkened. Hind femur slightly clavate, hind tibia slender. Metatibial spur rather long, blunt. Fore femur length $1.8-2.4 \mathrm{~mm}$ $(\mathrm{N}=9)$, width $0.69-0.86 \mathrm{~mm}(\mathrm{~N}=7)$, fore tibia length $1.7-2.4 \mathrm{~mm}(\mathrm{~N}=11)$, width $0.46-0.47 \mathrm{~mm}(\mathrm{~N}=2)$, hind femur length 2.6-3.6 mm ( $=11)$, width $0.54-0.76 \mathrm{~mm}(\mathrm{~N}=9)$, hind tibia length $2.7-3.9 \mathrm{~mm}$ $(\mathrm{N}=11)$, hind first tarsomere $1.0-1.2 \mathrm{~mm}(\mathrm{~N}=5)$.

Abdomen: Length 6.1-9.2 mm ( $\mathrm{N}=15)$, width 2.5-2.8 mm (N=6). Blackish brown.

Terminalia (fig. 206): Cercus slender, rounded. It may appear to have two segments but this is probably an artefact.

There is also a specimen in FSL (FSL 391912) labeled as a paratype of B. spectabilis. This specimen, however, has a slender fore tibia and a distinctive vein $\mathrm{R}_{2+3}$ and belongs in the genus Plecia. In ETHZ there is a female specimen labeled "Bibio spectabilis Heer" (ETHZ Pl. I 855) from the Miocene site of Öhningen. The specimen actually belongs to Bibio moestus (Skartveit \& Pika 2014), and the name Bibio spectabilis Heer was apparently never formally published and so does not affect the nomenclature of this species. Using the dark-banded wings as an identification character is problematic since the vast majority of the specimens from Aix-en-Provence have no preserved wing pigmentation.

\section{Genus Bibiodes Coquillett, 1904}

Bibiodes provincialis sp.n. (figs. 69-70, 207-211) 
Material examined: Holotype (male) MNHN Aix 1079. The specimen is preserved lying on the side, but with abdomen twisted so that the terminalia can be seen as if from dorsal view. Paratypes: males: MNHN Aix 62, 676. Females: MNHN Aix 23, 543, 959.

Localities: Aix-en-Provence

Diagnosis: A medium-small (5.5-7.5 mm) bibionid, though relatively large for the genus. Antenna stout, flagellum possibly 5 -segmented. Hind tibia strongly clavate. Male gonostyli are simple and digitiform.

\section{Description:}

Male: Total length 5.5-7.0 $\mathrm{mm}(\mathrm{N}=3)$. The specimens have head and thorax brown, legs and abdomen pale brown, no pigmentation preserved in wings.

Head (fig. 208): Length 0.97-1.24 mm (N=3). Ocellar triangle tall and prominent. Complex eye without conspicuous pile, lower section prominent. Antenna stout, flagellum possibly 5-segmented, $0.63 \mathrm{~mm}$ long $(\mathrm{N}=1)$.

Thorax: Length 2.0-2.8 mm (N=3). Brown.

Wing: Length 4.3-5.8 mm (N=3), width 1.7-2.5 mm (N=2), length/width $=2.37-2.50(\mathrm{~N}=2))$. Apparently hyaline. Few details discernible in specimen at hand. Wing vein measurements in Table 5. Legs: Protibia with long spine. Hind femur and tibia strongly clavate. Hind tarsus swollen. Length of fore tibia $1.2-1.5 \mathrm{~mm}(\mathrm{~N}=2)$, fore first tarsomere $0.9 \mathrm{~mm}(\mathrm{~N}=1)$, hind femur 1.6-2.5 $\mathrm{mm}(\mathrm{N}=3)$, width $0.31-0.50 \mathrm{~mm}(\mathrm{~N}=3)$, hind tibia $1.5-2.4 \mathrm{~mm}(\mathrm{~N}=3)$, width $0.37-0.50 \mathrm{~mm}(\mathrm{~N}=3)$, hind first tarsomere $0.7 \mathrm{~mm}(\mathrm{~N}=1)$.

Abdomen: Length 3.8-4.0 mm (N=3). Pale brown.

Terminalia (fig. 211): Hypopygium width $0.57-0.86 \mathrm{~mm}(\mathrm{~N}=2)$. Epandrium bilobate, lobes rounded, indentation between them widely U-shaped. Gonostylus length $0.31 \mathrm{~mm}(\mathrm{~N}=1)$, digitiform.

Female (fig. 209): Total length 6.0-7.5 mm (N=3). All specimens are preserved as relief impressions with little or no preserved colouration.

Head: Length $0.81-0.83 \mathrm{~mm}(\mathrm{~N}=1)$, width $0.81-0.93 \mathrm{~mm}(\mathrm{~N}=2)$. Relatively short and broad. Ocellar triangle prominent. Complex eye rounded, length approximately half length of head. Antenna stout, flagellum $0.5 \mathrm{~mm}$ long $(\mathrm{N}=1)$, possibly 5 -segmented.

Thorax: Brown. Length 1.8-2.5 mm (N=3), width $1.9 \mathrm{~mm}(\mathrm{~N}=1)$.

Wing (fig. 207): Length 5.2-6.5 mm (N=3), width 2.0-2.7 mm ( $=3)$, length/width $=2.11-2.64$ $(\mathrm{N}=3)$. Veins fine, Costa extends slightly beyond apex of $\mathrm{R}_{4+5}$. Veins $\mathrm{R}_{\mathrm{s}}$ and $\mathrm{M}$ are merged for a distance about twice the length of basal section of Rs. Pterostigma not apparent. Wing vein measurements in Table 5.

Legs (fig. 210): Protibia with ventral tubercle and long spine. Hind tibia clavate, hind tarsus swollen. Fore femur length $1.3-1.6 \mathrm{~mm}(\mathrm{~N}=2)$, width $0.4-0.7 \mathrm{~mm}(\mathrm{~N}=2)$. Fore tibia length $1.2-1.8 \mathrm{~mm}$ $(\mathrm{N}=2)$. Hind femur length $1.7-2.3 \mathrm{~mm}(\mathrm{~N}=2)$, width $0.37-0.49 \mathrm{~mm}(\mathrm{~N}=2)$, hind tibia length 1.6-2.1 $\mathrm{mm}(\mathrm{N}=2)$, width $0.29-0.39 \mathrm{~mm}(\mathrm{~N}=2)$. Hind first tarsomere $0.5-0.8 \mathrm{~mm}(\mathrm{~N}=2)$.

Abdomen: Cylindrical. Length 3.9-4.7 mm (N=3), width 1.8-2.1 mm (N=2).

Terminalia: Not clearly visible in any of the specimens at hand. 


\section{Bibiodes sp. (Fig. 212)}

This species is represented by a single female specimen.

Material: NMB F2136 (1 female)

\section{Localities: Kleinkembs}

Diagnosis: The single female specimen is not well enough preserved to be reliably separated from $B$. provincialis, but the antenna appears to be more slender, and the section where veins Rs and $\mathrm{M}$ are fused appears to be shorter than in B. provincialis.

\section{Description:}

Male unknown

Female: Total length $6.5 \mathrm{~mm}(\mathrm{~N}=1)$.

Head: Length $0.7 \mathrm{~mm}(\mathrm{~N}=1)$. Black. Complex eye relatively small. Ocellar tubercle tall and prominent. Antenna not well preserved but appears to be relatively slender.

Thorax: Length $2.2 \mathrm{~mm}(\mathrm{~N}=1)$. Black. Scutum strongly developed, relatively flat. Haltere dark reddish brown.

Wing: Length $6.0 \mathrm{~mm}$, width $2.1 \mathrm{~mm}$, length/width $=2.92(\mathrm{~N}=1)$. Hyaline, veins yellowish, pterostigma oval, light brown, distinctive. Costa extends slightly beyond apex of $\mathrm{R}_{4+5}$. Subcosta fine and indistinctive, humeral vein probably present. Radial sector and $\mathrm{M}$ merged for a short distance. $\mathrm{M}$ and $\mathrm{CuA}$ with relatively short stems and long forks. Wing vein measurements in Table 5 .

Legs: Blackish-brown, presumably black in life, robust. Fore femur length $1.4 \mathrm{~mm}$, width $0.62 \mathrm{~mm}$, fore tibia length $1.5 \mathrm{~mm}$, with short, stout spur, less than one third as long as spine. Hind femur length $1.9 \mathrm{~mm}$, width $0.44 \mathrm{~mm}$, stout throughout, hind tibia length $2.0 \mathrm{~mm}$, width $0.27 \mathrm{~mm}$, slender, first tarsomere slender.

Abdomen: Chestnut-brown, rather stout, cylindrical. Length $3.8 \mathrm{~mm}(\mathrm{~N}=1)$.

Terminalia: The tergites of the last three segments are apparently missing, so the internal structures are uncovered. Genital fork and two spermathecae are clearly visible, but a third spermatheca may be partly hidden.

\section{Genus Dilophus Meigen, 1803}

Dilophus luteipennis (Theobald, 1937) comb.n. (Fig. 71, 213-214)

Bibio luteipennis Théobald, 1937: 333.

Material examined: Holotype (female) of Bibio luteipennis FSL 391913 (A 16). Paratype (female) of Bibio luteipennis FSL 391914 (A 86).

Localities: Aix-en-Provence. 
Diagnosis: A medium-sized Dilophus, 5.5-6.0 mm. Mesonotum black, pleurae yellowish, legs yellowish-brown with black tarsi. Protibia possibly with three mesal spines. Wing hyaline with small, dark brown pterostigma.

\section{Redescription:}

Male unknown. There are several male Dilophus specimens from Aix-en-Provence in MNHN, but it is highly uncertain if these belong to D. luteipennis.

Female: Total length 5.5-6.0 mm (N=2).

Head (fig. 214): Length $0.7 \mathrm{~mm}(\mathrm{~N}=1)$. Black. Antenna short and stocky, segments not possible to count in the specimens at hand.

Thorax: Length 1.3-1.5 mm ( $\mathrm{N}=2)$, width $1.4 \mathrm{~mm}(\mathrm{~N}=1)$. Mesonotum black, pleura brownish-yellow. Spine rows not possible to see in the specimens at hand.

Wing (fig. 213): Length 4.0-4.6 mm ( $\mathrm{N}=2)$, width 1.5-1.8 $\mathrm{mm}(\mathrm{N}=2)$. Hyaline, pterostigma small, oval and dark brown, R-veins dark brown, posterior veins colourless. Costa extends to apex of $\mathrm{R}_{4+5}$.Crossvein $\mathrm{R}-\mathrm{M}$ slightly longer then basal part of $\mathrm{R}_{\mathrm{s}}$. Wing vein measurements in Table 5.

Legs: Yellowish-brown, tarsi black. Protibia rather stout, possibly with three mesal spines. Hind femur $1.3 \mathrm{~mm}$, hind tibia $1.2 \mathrm{~mm}(\mathrm{~N}=1)$.

Abdomen: Length 3.7-4.4 mm (N=2), width $1.5 \mathrm{~mm}(\mathrm{~N}=1)$. Cylindrical, dark brown.

Terminalia: Cerci rather long, apically blunt, slightly hooked.

\section{Further Dilophus specimens}

Specimens belonging to the genus Dilophus are scarce but present from many of the localities. Unfortunately, important diagnostic features such as the shape of male terminalia, antennae and protibiae are rarely preserved, thus it is not advisable to describe new species based on this material.

The genus has been noted from the following localities:

Céreste: EEFN F 312 (male, 6.7 mm). This specimen was listed by Théobald (1937: 404) as Bibio detractus.

Chadrat: MNHN Chadrat P10 (female, $8 \mathrm{~mm}$ ).

Monteils: MNHN Monteils 148 (male, $4.3 \mathrm{~mm}$ ), Monteils 159 (female, $8 \mathrm{~mm}$ ).

Bois d'Asson: RGL Bois d'Asson 43 (female, $6.0 \mathrm{~mm}$ ).

Aix-en-Provence: MNHN Aix 18 (female, $10 \mathrm{~mm}$ ), Aix 31 (male, $5.0 \mathrm{~mm}$ ), Aix 34 (male, $6.5 \mathrm{~mm}$ ), Aix 491 (male, thorax length $1.2 \mathrm{~mm}$ ), Aix 797 (male, $6.2 \mathrm{~mm}$ ), Aix 1079 (male, $5.5 \mathrm{~mm}$ ).

Kleinkembs: NMB F2110 (male, $5.5 \mathrm{~mm}$ ), F2246 (male, $5.5 \mathrm{~mm}$ ).

\section{Unrecognizable species}

A number of species described from French Oligocene deposits is based on material which is too poorly preserved to be recognizable at the species level. We have examined the types of the following and they should be reclassified as follows:

Bibio disjectus Théobald, 1937: 334. Nomen dubium. Holotype: FSL 391916. The type specimen is a male specimen, $8.5 \mathrm{~mm}$ long. It is possibly the same as Bibio major, but since neither head, legs nor wings are well preserved in the type specimen, the species cannot be recognized. 
Plecia larteti (Oustalet, 1870): 137 [Bibio]. Nomen dubium. Holotype: MNHN R 06667. Female specimen, total length $6.7 \mathrm{~mm}$. The specimen is too poorly preserved to be recognizable at the species level, but the wing venation reveals it is a Plecia.

Plecia painvini (Meunier, 1915): 16. [Bibio]. Nomen dubium. Probable holotype: EM 70000, temporarily stored in FSL. The specimen fits the photo of the holotype given in Meunier's paper (Fig. 18) and also fits the measurements given by Meunier perfectly. It is a poorly preserved Plecia female.

Plecia nigrescens Oustalet, 1870: 115. Nomen dubium. Syntypes: MNHN R 06691 and R 06694. Two male specimens. Wings and legs are missing in both specimens, hypopygium not visible. It is hard to ascertain what these specimens really are, they belong to Nematocera but we are not able to classify them further.

Plecia retracta Théobald, 1937: 341-342. Nomen dubium. Holotype: FSL 391936. A female specimen, part of the wing visible but no systematically important characters possible to see. Belongs in the genus Plecia.

Protomyia adusta Oustalet, 1870: 145. Nomen dubium. Holotype: MNHN. A very weak impression, is a bibionid but not further classifiable.

Protomyia formicoides Oustalet, 1870: 150. Nomen dubium. Holotype: MNHN R 06681. Female specimen, total length approx. $6.5 \mathrm{~mm}$. A very weak impression, not recognizable to genus but it is in all likelihood a bibionid.

Protomyia fusca Oustalet, 1870: 145. Nomen dubium. Holotype: MNHN R 06672. Female specimen, very vague impression, wings and legs missing. Is probably a bibionid.

Plecia joannis (Oustalet, 1870): 143. [Protomyia]. Nomen dubium. Holotype: MNHN R 06690.

Female specimen, approx. $8 \mathrm{~mm}$ long, very weak impression. A short $\mathrm{R}_{2+3}$ shows it to be a Plecia, but otherwise unrecognizable.

Plecia longipennis (Oustalet, 1870): 141. [Protomyia]. Nomen dubium. Holotype: MNHN R 06673. Female specimen, total length $6.1 \mathrm{~mm}$.

Plecia rubescens (Oustalet, 1870): 149. [Protomyia]. Nomen dubium. Holotype: MNHN R 07026. Female specimen, approx. $8 \mathrm{~mm}$ long. The specimen is bright rusty-red due to impregnation with iron. It is a Plecia but is not further recognizable.

Plecia sauvagei (Oustalet, 1870): 146. [Protomyia]. Nomen dubium. Holotype: MNHN R 06680. A rather large Plecia female, total length $9.6 \mathrm{~mm}$. Not recognizable.

\section{Species for which we have not found type material}

Judging from the original descriptions and the accompanying drawings, probably none of these species described by Oustalet (1870) are recognizable at the species level, though for most it is possible to place them tentatively to genus. All these names should be considered nomina dubia unless the type specimens are found at some time in the future.

Penthetria vaillantii Oustalet, 1870: 112-113. The species was drawn with an unbranched $\mathrm{R}_{4+5}$ (Oustalet 1870 Pl. 3, Figs. 1-2). Penthetria specimens with unbranched $\mathrm{R}_{4+5}$ are known (Skartveit 2009), but this is highly un-typical of the genus. It is not clear from Oustalet's drawings if the species belongs in Bibionidae. Identification: Nematocera indet.

Plecia angustiventris Théobald, 1937: 135. Holotype supposed to be in Marseille, presumably lost. The photo given by Théobald (1937, Pl. II, Fig. 4) shows a female specimen with a short $\mathrm{R}_{2+3}$. The species belongs in the genus Plecia. 
Plecia dumasi Théobald, 1937: 138. Holotype supposed to be in Nîmes, presumably lost. The illustration (Théobald 1937, Pl. XI, Fig. 2) accompanying the original description shows a typical female Plecia.

Plecia pallida Oustalet, 1870: 118-119. Oustalet (1870, Pl. 3. Figs. 11-13) depicted three male specimens. No systematically important characters can be seen in the drawings, but the habitus suggests the species is correctly placed in Plecia.

Bibio alacris Oustalet, 1870: 127. Oustalet (1870, Pl. 3, Fig. 15) depicted a slender specimens with thin anterior legs - certainly not a Bibio species. Judging from the habitus the species probably belongs in genus Penthetria.

Bibio cylindratus Oustalet, 1870: 133. Oustalet (1870, P1. 4, Fig. 12) depicted a bibionid female. The wing venation is not apparent from the drawing, but the habitus suggests it is either Penthetria or Plecia, most likely the latter.

Bibio elongatipennis Théobald, 1937: 141. Holotype supposed to be in Marseille, presumably lost. The photo (Théobald 1937, Pl. II, Fig. 13) given in the original description shows a slender female specimen which clearly belongs in the genus Penthetria.

Bibio gigas Oustalet, 1870: 122. Oustalet (1870, Pl. 4, Figs. 1-4) drew four female specimens, all poorly preserved. Their long, slender bodies suggest it should be placed in genus Penthetria, as does the large size of the specimens.

Bibio macer Oustalet, 1870: 126 (nec Loew, 1871). Oustalet (1870, Pl. 4, Fig. 6) drew a female specimen without wings, but with slender legs. The elongate body shape suggests it belongs in Penthetria.

Bibio maceratus Théobald, 1937: 142-143. Holotype supposed to be in Marseille, presumably lost. The illustration given by Théobald (1937, Pl. II, Fig. 12) shows a slender female specimen, probably belonging to the genus Penthetria. Judging from the illustrations there is a very high likelihood that $B$. elongatipennis and B. maceratus are synonyms.

Bibio minor Oustalet, 1870: 134. Oustalet (1870, Pl. 3, Fig. 16) drew a slender, female specimen. No vein $\mathrm{R}_{2+3}$ was indicated, but the slender habitus and the vertical R-M suggests it belongs in Penthetria.

Bibio nigripennis Théobald, 1937: 286, nec Brunetti, 1913. Holotype supposed to be in Marseille, presumably lost. One specimen labeled as this species in MHNM is not the one illustrated by Théobald, and is actually a chironomid. The species name is a primary junior homonym of Bibio nigripennis Brunetti, 1913 and thus invalid. The illustration (Théobald 1937, Pl. XX, Fig. 5) shows a female specimen which, although no vein $\mathrm{R}_{2+3}$ is apparent, clearly belongs in the genus Plecia. We propose the name Plecia theobaldi nom.n. as a replacement name for this species.

Bibio robustus Oustalet, 1870: 128. Oustalet (1870, Pl. 4, Figs. 7-9) drew three female specimens in poor condition. No vein $\mathrm{R}_{2+3}$ is apparent in the drawings, but the habitus will place the species in genus Plecia or Penthetria, most likely the former.

\section{Discussion}

Bibionid flies make up a morphologically rather homogenous group and differences between species tend to be slight in the recent fauna. Thus, new species in this group should not be described based on fragmentary fossil material except possibly for species that possess some distinctive and unusual morphological traits. As a minimum, specimens should be available which show the wing venation and the male terminalia (particularly epandrium and gonostyles) and preferably the antennae. In the 
Bibioninae, fore and hind legs should also be available for study, at least the tibiae which are very useful in this subfamily. The appearance of the genital structures are highly dependent upon the position in which they have been fossilized. Since compression fossils cannot be rotated, this is highly problematical. In the present material we were able to deduce the shape of the male gonostylus because we had access to a very large number of specimens in which this was fixed in various positions. With a smaller number of specimens, it is easy to interpret the various positions as distinctive shapes and classify them as different species.

The majority of named fossil bibionids have been described based on specimens which do not fulfil these requirements, in particular most are based on female specimens, and connecting male and female specimens has rarely been attempted. The synonymies we have suggested are tentative in the sense that it is always possible that two nominal species could be distinguished if perfectly preserved specimens, showing all relevant characters, were available. However, we believe that a conservative approach should be followed in paleontology, whereby sound evidence is needed to justify the treatment of two taxa as different. In the absence of such evidence they should, for simplicity, be regarded as the same. This approach may lead to over-estimates of the longevity of species when sound evidence for distinguishing specimens of different age cannot be found.

Except for the Kleinkembs locality that is situated in the northern part of France, all the studied outcrops are distributed in the relatively narrow area in the Centre and South-East of France. Therefore they are of interest for a study of the evolution of the bibionid faunas (see Fig. xxx, map).

In the present material, a distinctive change in the bibionid faunas during the Oligocene is obvious. The oldest localities at Célas, Monteils and Camoins harbour a distinctive, Early Oligocene fauna including Penthetria graciliventris, Penthetria longiventris and Bibio celasensis. Most of the species found in the locality of Kleinkembs are unique to this site, however there are shared species both with older (Plecia foersteri shared with Célas) and presumably younger (Penthetria lugens, Plecia lapidaria) sites. The most distinctive element of the middle Oligocene sites such as Bois d'Asson, Corent and Céreste are the abundant species Plecia livida and Bibio major, the former however also occur in Aix-en-Provence. The youngest site of Aix-en-Provence has several unique species: Plecia morio, Bibio aquaesextiae, Bibio curtisii, Bibio spectabilis, Bibiodes provincialis and Dilophus luteipennis. At least three of the species found in Aix also occur in the similar-age site of Rott, Germany and one (Plecia lygaeoides) in the younger site of Radoboj, Croatia.

Fossil bibionid flies are potentially useful for the reconstruction of past environments, in particular climate (Collomb et al. 2008). But if such attempts are to be worthwhile, it is essential that the taxonomy is realistic. The estimates of Collomb et al. (2008) were based on the frequencies of different bibionid genera (based on recently collected material), which makes sense since the modern bibionid genera have to a large extent different climate requirements. In working with the present revision we have found that a large fraction of the nominal species have been placed in the wrong genus, which will compromise the use of the ancient described species to make inferences about past climates. On the other hand, a realistic classification of fossil bibionids may open new opportunities to use these abundant fossils in the study of past environments. At present, the somewhat ambiguous delimititation of the genera Penthetria and Plecia causes problems for climate reconstructions since these two genera have different climate preferences.

\section{Acknowledgements}

We are very thankful to the curators of the various museums for giving us access to and helping us find the material studied. Curators Emmanuel Robert, FSL, Lyon, Milena Pika-Biolzi, ETHZ, Zürich, Walter Etter, NMB, Basel, Sylvie Pichard, MNHM, Marseille, Jean Philip and Bertrand Martin-Garin, 
MPP, Marseille, Dino Frey and Wolfgang Munk, SMNK, Karlsruhe, Claire Mellish, NHMUK, London, Oleg Mandic, NHMW, Vienna and Martin Gross, SLJG, Graz all helped with this. Andrew Ross, National Museum of Scotland, Edinburgh gave us valuable help in locating and interpreting some type material. Biodiversity Heritage Library (http://www.biodiversitylibrary.org/) has proved to be a very valuable source for older literature, which greatly aided this work.

\section{References}

Brongniart, C.J.E. (1876) Observations sur un insecte fossile de la famille des diptères trouvé à Chadrat (Auvergne) (Protomyia oustaleti). Annales des Sciences Géologiques 7, 1-2.

Brongniart, C.J.E. (1878) Note rectificative sur les espèces de Bibionidae fossiles du genre Plecia. Bulletin des Séances de la Société entomologique de France 1878, XLVII-XLVIII.

Brunetti, E., 1913. New and interesting Diptera from the Eastern Himalayas. Records of the Indian Museum 9: 255-277.

Buckland, W. (1837) Geology and Mineralogy Considered with Reference to Natural Theology. Vol. II. Carey, Lea and Blanchard, Philadelphia. 132 pp. +69 plates.

Collomb, F.-M., Nel, A., Fleck, G. \& Waller, A. (2008) March flies and European Cenozoic palaeoclimates (Diptera: Bibionidae). Annales de sociéte entomologique de France (nouvelle serie) 44, 161-179.

Coquillett, D.W. (1904) New North American Diptera. Proceedings of the Entomological Society of Washington 6: 166-192.

Evenhuis, N.E. (1994) Catalog of the fossil flies of the world. Backhuys Publishers, Leiden. 600 pp.

Fabricius, J.C. (1787) Mantissa Insectorum sistens species nuper detectas adiectis synonymis, observationibus, descripitionibus, emendationibus. Tom. II. Christ. Gottl. Proft, Hafniae (Copenhagen). $382 \mathrm{pp}$.

Gentilini, G. (1993) New species of Plecia from the Oligocene of Southeastern France. Bollettino della Societa Entomologica Italiana 125, 157-165.

Geoffroy, E.L. (1762) Histoire abrégee des insectes qui se trouvent aux environs de Paris. 2. Durand, Paris, 690 pp.

Haenni, J.-P. \& Merz, B. (2000) Morphology and terminology of adult Diptera (other than terminalia). In: Papp, L. \& Darvas, B. (eds.) Contributions to a Manual of Palaearctic Diptera - Vol. 1. General and Applied Dipterology. Science Herald, Budapest. pp. 21-51.

Handlirsch, A. (1908) Die fossilen Insekten und die Phylogenie der rezenter Formen. Ein Handbuch für Paläontologen und Zoologen. Textband. W.Engelmann, Leipzig.

Heer, O. (1849) Die Insektenfauna der Tertiärgebilde von Oeningen und von Radoboj in Croatien. Zweite Abtheilung: Heuschrecken, Florfliegen, Aderflügler, Schmetterlinge und Fliegen. Neue Denkschriften Allgemeinen Schweizerischen Gesellschaft für die Gesamten Naturwissenschaften, Zürich 11, 1-264.

Heer, O. (1856) Ueber die fossilen Insekten von Aix in der Provence. Vierteljahrsschrift der Naturforschenden Gesellschaft in Zürich 1, 1-40. 
Heer, O. \& Gaudin, C.-T. (1861) Recherches sur le climat et la végétation du pays tertiaire. Wurster, J. and Winterthur, C., Cherbuliez, Geneva and Paris. xxii +220 pp.

Heyden, C. von \& Heyden, L. von (1865) Bibioniden aus der Rheinischen Braunkohle von Rott. Palaeontographica 14, 19-30.

Linnaeus, C. (1758) Systema naturae per regna tria naturae, secundum classes, ordines, genera, species, cum caracteribus, differentiis, synonymis, locis. Tomus I. Editio decima, reformata. L. Salvii, Holmiae (Stockholm), 824 pp.

Loew, H. (1871) Systematische Beschreibung der bekannten europäischen zweiflügeligen Insecten, von Johann Wilhelm Meigen. Neunter Theil oder dritter Supplementband. Beschreibungen europäische Dipteren. Zweiter Band. Halle.

Meigen, J.W. (1803) Versuch einer neuer GattungsEintheilung der europäischen zweiflügeligen Insecten. Magazin für Insektenkunde (Illiger) 2: 259-281.

Meunier, F. (1915a) Nouvelles recherches sur quelques insectes des plâtrières d'Aix en Provence. Verslag van de Gewone Vergaderingen der Wis- en Natuurkundige Afdeeling der Koninklijke Akademie van Wetenschappen te Amsterdam 18, 1-17.

Meunier, F. (1915b) Über einige fossile Insekten aus den Braunkohlenschichten (Aquitanien) von Rott (Siebengebirge). Zeitschrift der Deutschen Geologischen Gesellschaft (A) 67, 205-217.

Murchison, R.I. \& Lyell, C. (1829) On the Tertiary formation of Aix, in Provence, including the Coalfield of Fuveau, with a description of fossil insects, shells and plants, contained therein. The Edinburgh new philosophical journal October 1829, 287-297.

Oustalet, E. (1870) Recherches sur les insectes fossiles des terrains tertiaires de la France. Annales des Sciences Géologiques 2, 1-178.

Oustalet, E. (1872) Sur l'empreinte d'une aile de Diptère. Bulletin de la Société Philomatique de Paris $1872,161-163$.

Rice, H.D.A. (1959) Fossil Bibionidae (Diptera) from British Columbia. Dept. of Mines and Technical Survey, Canada, Bulletin 55. 35 pp.

Skartveit, J. (2009) Fossil Bibionoidea (Diptera: Hesperinidae and Bibionidae) from Baltic amber. Studia dipterologica $15,3-42$.

Skartveit, J. \& Pika, M. (2014) Revision of Bibionidae (Diptera) named by Oswald Heer from the Miocene of Öhningen, Southern Germany. Mitteilungen der schweizerischen entomologischen Gesellschaft 87, 103-134.

Statz, G. (1943) Neue Dipteren (Nematocera) aus dem Oberoligocän von Rott. Palaeontographica 45, $1-65+$ plates

Théobald, N. (1937) Les insectes fossiles des terrains oligocènes de France. Bulletin mensuel (Mémoires) de la Sociéte des Sciences de Nancy 1, 1-473.

Unger, F. (1842) Fossile Insecten. Nova acta physico-medica Academiae Caesareae LeopoldinoCarolinae Naturae Curiosum 19: 415-428.

Wedmann, S. (1998) Taphonomie der Bibionidae (Insecta: Diptera) aus der oberoligozänen Fossillagerstätte Enspel (Westerwald, Deutschland). Neues Jahrbuch für Geologie und Paläeontologie Monatshefte 1998: 513-528. 
Wiedemann, C.R.W. (1828) Aussereuropäische zweiflügelige Insekten. Aus Fortsetzung des Meigensche Werkes. Erster Theil. Schulz,Hamm, 608 pp. Bibionidae pp.72-81.

Wootton, R.J. \& Ennos, A.R. (1989) The implications of function on the origin and homologies of the dipterous wing. Systematic Entomology 14, 5+7-520. 


\section{Legends to figures}

Fig. 1. Penthetria claripennis, female. Holotype of Plecia claripennis Théobald, NMB F1382.

Fig. 2. Penthetria claripennis, male. Holotype of Plecia variegata Théobald, NMB F1405.

Fig. 3. Pentehtria claripennis, male. Holotype of Plecia varia Théobald, NMB F1407.

Fig. 4. Penthetria claripennis, male. Holotype of Plecia difficilis Théobald, NMB F1408.

Fig. 5. Penthetria claripennis, male. Holotype of Plecia graciosa Théobald, NMB F1409.

Fig. 6. Penthetria gigantea, female. Holotype of Plecia gigantea Théobald, NMB F1380.

Fig. 7. Penthetria gigantea, female. Holotype of Plecia crassiventris Théobald, NMB F1381.

Fig. 8. Penthetria gigantea, male. Holotype of Plecia miegi Théobald, NMB F1399.

Fig. 9. Penthetria gigantea, male. Holotype of Plecia luteipennis Théobald, NMB F1402.

Fig. 10. Penthetria graciliventris. MNHN Monteils 35.

Fig. 11. Penthetria longiventris, female. Paratype of Plecia superba Théobald, FSL 391901.

Fig. 12. Penthetria luberonica, male. Holotype of Penthetria luberonica sp.n., ECL 140.048/2.

Fig. 13. Penthetria luberonica, female. ECL 140.046.

Fig. 14. Penthetria lugens, female. Holotype of Protomyia lugens Oustalet, MNHN R 06666.

Fig. 15. Penthetria lugens, female. NMB F2410.

Fig. 16. Penthetria nervisinuata, female. Holotype of Plecia nervisinuata Théobald, FSL 391925.

Fig. 17. Penthetria nervisinuata, female. ETHZ, labeled 164.

Fig. 18. Penthetria subterranea, male. Holotype of Plecia subterranea Théobald, NMB F1404.

Fig. 19. Plecia assonensis, male. MNHN Bois d'Asson 81.

Fig. 20. Plecia bucklandi, female. Specimen identified as Protomyia bucklandi Heer by Heer, ETHZ, no specimen number.

Fig. 21. Plecia bucklandi, female. Syntype of Protomyia elegans Heer, NHMUK In 39832. Image reproduced with permission from NHMUK.

Fig. 22. Plecia bucklandi, female. Syntype of Protomyia gracilis Heer, NHMUK In 38765. Image reproduced with permission from NHMUK.

Fig. 23. Plecia bucklandi, female. Syntype of Bibio edwardsii Oustalet, MNHN R 06671.

Fig. 24. Plecia bucklandi, female. Holotype of Plecia dilatativentris Théobald, FSL 391924.

Fig. 25. Plecia foersteri, female. Holotype of Plecia försteri Théobald, NMB F1390

Fig. 26. Plecia foersteri, female. Holotype of Plecia splendida Théobald, NMB F1396. 
Fig. 27. Plecia foersteri, female. Holotype of Plecia oviformis Théobald, NMB F1384.

Fig. 28. Plecia foersteri, female. "Hypotypoide" of Plecia rubescens (Oustalet) sensu Théobald, NMB F1395/1.

Fig. 29. Plecia conica, male. Holotype of Plecia conica Théobald, NMB F1398.

Fig. 30. Plecia conica, male. MNHN Dauphín 12.

Fig. 31. Plecia inflata, female. Holotype of Plecia inflata Oustalet, NMB F1495.

Fig. 32. Plecia lapidaria, female. Syntype of Plecia lapidaria Heyden \& Heyden, NHMUK In 58813. Image reproduced with permission from NHMUK.

Fig. 33. Plecia lapidaria, female. NMB F2406.

Fig. 34. Plecia larguensis, male. RGL Bois d'Asson 7.

Fig. 35. Plecia larguensis, male. RGL Dauphín 30.

Fig. 36. Plecia livida, female. Lectotype of Protomyia livida Heer, NHMUK In 39854. Image reproduced with permission from NHMUK.

Fig. 37. Plecia livida, male. Syntype of Protomyia brevipennis Heer, NHMUK In 39830. Image reproduced with permission from NHMUK.

Fig. 38. Plecia livida, male. Holotype of Plecia acutiventris Théobald, MNHN Ex. 169.

Fig. 39. Plecia livida, female. Holotype of Plecia goreti Théobald, MNHN B 24516.

Fig. 40. Plecia livida, female. Paratype of Plecia guineri Théobald, MNHN B 24415.

Fig. 41. Plecia livida, male. Holotype of Plecia maculiventris Théobald, MNHN B 24523.

Fig. 42. Plecia lygaeoides, female. Holotype of Protomyia lygaeoides Heer, SLJG 77465.

Fig. 43. Plecia lygaeoides, male. Holotype of Protomyia gracilenta Heer, BMNH In 58805. Image reproduced with permission from $\mathrm{BMNH}$.

Fig. 44. Plecia lygaeoides, male. Holotype of Bibio detractus Théobald, FSL 391921.

Fig. 45. Plecia maimensis, male. RGL Dauphín 122.

Fig. 46. Plecia maimensis, female. RGL Bois d'Asson 4.

Fig. 47. Plecia morio, female. NHMUK In 39831. Image reproduced with permission from NHMUK.

Fig. 48. Plecia morio, female. Syntype of Protomyia collossea Heyden \& Heyden, NMHUK In 58789. Image reproduced with permission from NHMUK.

Fig. 49. Plecia rhenana, female. Syntype of Plecia rhenana Heyden \& Heyden, NHMUK In 38798. Image reproduced with permission from NHMUK.

Fig. 50. Bibio aquaesextiae, male. Holotype of Bibio aquaesextiae sp.n., MNHN Aix 152.

Fig. 51. Bibio baumbergeri, female. Holotype of Bibio baumbergeri Théobald, NMB F456. 
Fig. 52. Bibio baumbergeri, female. Holotype of Bibio parenti Théobald, NMB F1372.

Fig. 53. Bibio celasensis, male. Male specimen from syntype slab, MHNM.

Fig. 54. Bibio celasensis, female. Female specimen from syntype slab, MHNM.

Fig. 55. Bibio celasensis, female. Syntype of Bibio conformans Théobald, FSL 392012.

Fig. 56. Bibio curtisii, male. Lectotype of Bibio curtisii Heer, NHMUK In 39833. Image reproduced with permission from NHMUK.

Fig. 57. Bibio curtisii, male. Counterpart of lectotype, NHMUK In 39855. Image reproduced with permission from NHMUK.

Fig. 58. Bibio curtisii, female. Possible type of Bibio martinsi Heer, ETHZ S.N. 131.

Fig. 59. Bibio curtisii, female. Holotype of Bibio romani Théobald, FSL 391915.

Fig. 60. Bibio curtisii, female. Paratype of Bibio serresi Théobald, FSL 391905.

Fig. 61. Bibio curtisii, sex uncertain. Specimen identified as Bibio bimaculatus (Heer? Status uncertain), ETHZ 137.

Fig. 62. Bibio dislocatus, female. Holotype of Bibio dislocatus Théobald, NMB F1378.

Fig. 63. Bibio dislocatus, female. Holotype of Bibio consolida Théobald, NMB F1379.

Fig. 64. Bibio dislocatus, female. Holotype of Plecia macroventris Théobald, NMB F1383.

Fig. 65. Bibio major, male. Syntype of Plecia major Oustalet, MNHN R 06693.

Fig. 66. Bibio major, female. Syntype of Bibio ungeri marginatus, MNHN R 07253.

Fig. 67. Bibio major, male. RGL Céreste 316.

Fig. 68. Bibio spectabilis, female. Holotype of Bibio spectabilis Théobald, FSL 391911.

Fig. 69. Bibiodes provincialis, male. Holotype of Bibiodes provincialis sp.n., MNHN Aix 1079.

Fig. 70. Bibiodes provincialis, female. MNHN Aix 959.

Fig. 71. Dilophus luteipennis, female. Holotype of Bibio luteipennis Théobald, FSL 391913. 
Scale on all drawings $=1 \mathrm{~mm}$.

Figs. 72-77 Penthetria claripennis (Théobald, 1937).

Fig. 72. Male, wing. NMB F1416/1.

Fig. 73. Male, terminalia, ventral. NMB F1416/3.

Fig. 74. Male, terminalia, dorsal. NMB F1416/6.

Fig. 75. Male, terminalia, lateral. NMB F1419/4.

Fig. 76. Female, wing. NMB F1382.

Fig. 77. Male, habitus: NMB F1418/1.

Figs. 78-80. Penthetria gigantea (Théobald, 1937)

Fig. 78. Male, wing. NMB F1399/1.

Fig. 79. Female, wing. NMB F1389/1.

Fig. 80. Male, terminalia, dorsal. NMB F1399/1.

Figs. 81-84. Penthetria graciliventris (Théobald, 1937)

Fig. 81. Male, wing. MNHN Monteils 57.

Fig. 82. Female, wing. MNHN Monteils 25.

Fig. 83. Male, terminalia, dorsal. MNHN Monteils 57.

Fig. 84. Male, head and thorax. MNHN Monteils 57.

Figs. 85-89. Penthetria longiventris (Théobald, 1937)

Fig. 85. Female, wing. MNHN Monteils 11.

Fig. 86. Male, head. MNHN Monteils 51.

Fig. 87. Male, terminalia, dorsal. MNHN Monteils 51.

Fig. 88. Female, head and thorax, lateral. MNHN Monteils 13.

Fig. 89. Female, head and thorax, dorsal. MNHN Monteils 145.

Figs. 90-94. Penthetria luberonica sp.n.

Fig. 90. Male, wing. ECL 140.050.

Fig. 91. Male, head and thorax. ECL 140.050.

Fig. 92. Male, terminalia. ECL 140.053/7.

Fig. 93. Female, wing. ECL 140.046.

Fig. 94. Female, head and thorax. ECL 140.053/5.

Figs. 95-97. Penthetria lugens (Oustalet, 1870)

Fig. 95. Female, wing. NMB F2410.

Fig. 96. Female, head and thorax, dorsal. FSL 391923.

Fig. 97. Female, terminalia, ventral. NMB F2413.

Figs. 98-92. Penthetria nervisinuata (Théobald, 1937) 
Fig. 98. Female, wing. ETHZ Aix 164.

Fig. 99. Female, head and thorax, dorsal. ETHZ Aix 164.

Fig. 100. Female (?), wing, RGL Céreste 192.

Fig. 101. Male, terminalia, dorsal. MNHN Aix 981.

Fig. 102. Male, wing. MNHN Aix 974.

Figs. 103-106. Penthetria subterranea (Théobald, 1937)

Fig. 103. Male, wing. NMB F1404.

Fig. 104. Male, terminalia, dorsal. NMB F1404.

Fig. 105. Male, terminalia, ventral. NMB F1414/1.

Fig. 106. Female, wing. EM 70010.

Figs. 107-109. Plecia assonensis Gentilini, 1993

Fig. 107. Male, wing. RGL Dauphín 33.

Fig. 108. Male, terminalia, dorsal. RGFL Bois d'Asson 81.

Fig. 109. Male, terminalia. RGL Bois d'Asson 34.

Figs. 110-113. Plecia bucklandi (Heer, 1849)

Fig. 110. Female, wing. ETHZ Aix 142.

Fig. 111. Male, wing. NHMUK In 39832.

Fig. 112. Female, head and thorax, lateral. ETHZ Aix 142.

Fig. 113. Female, head and thorax, dorsal. NHMUK In 39832.

Figs. 114-118. Plecia conica Théobald, 1937.

Fig. 114. Male, wing. NMB F2090.

Fig. 115. Male, habitus, lateral. RGL Dauphín 12.

Fig. 116. Male, habitus, dorsal. RGL Saint Maime 23'.

Fig. 117. Male, terminalia, dorsal. RGL Dauphín 12.

Fig. 118. Male, terminalia, lateral. NMB F1398/2.

Figs. 119-122. Plecia foersteri Théobald, 1937

Fig. 119. Female, wing. NMB F1411/22.

Fig. 120. Female, head and thorax, dorsal. NMB F2423.

Fig. 121. Female, terminalia, ventral. NMB F1411/13.

Fig. 122. Female, head and thorax, NMB F1390.

Figs. 123-125. Plecia inflata Oustalet, 1870

Fig. 123. Female, wing. NMB F2345. 
Fig. 124. Female, head and thorax, lateral. NMB F2215.

Fig. 125. Female, terminalia, dorsal. NMB F2344.

Figs. 126-128. Plecia lapidaria Heyden \& Heyden, 1865

Fig. 126. Female, wing. NHMUK In 58813.

Fig. 127. Female, head and thorax. NMB F1530.

Fig. 128. Female, terminalia. NMB F1565.

Figs. 129-131. Plecia larguensis Gentilini, 1993

Fig. 129. Male, wing. RGL Bois d'Asson 7.

Fig. 130. Male, head and thorax. RGL Bois d'Asson 7.

Fig. 131. Male, terminalia, dorsal. RGL Dauphín 182.

Figs. 132-143. Plecia livida (Heer, 1856)

Fig. 132. Male, wing. RGL Céreste 1002.

Fig. 133. Female, wing. RGL Céreste 476.

Fig. 134. Male, head and thorax, dorsal. RGL Céreste 1010.

Fig. 135. Female, head. RGL Céreste 734.

Fig. 136. Male, terminalia, dorsal. MNHN Aix 1354.

Fig. 137. Male, terminalia, dorsal. MNHN Aix 516.

Fig. 138. Male, terminalia, ventral. MNHN Aix 516.

Fig. 139. Male, terminalia, dorsal. RGL Céreste 1010.

Fig. 140. Male, terminalia, ventral, RGL Céreste 602.

Fig. 141. Male, aedeagus and associated structures, RGL Céreste 1010.

Fig. 142. Female, terminalia, ventral, RGL Céreste 499.

Fig. 143. Female, terminalia, dorsal, RGL Céreste 379.

Figs. 144-148. Plecia lygaeoides (Heer, 1849)

Fig. 144. Female, wing. FSL 391929.

Fig. 145. Male, head, NHMUK In 58805.

Fig. 146. Female, head. ETHZ.

Fig. 147. Male, terminalia, ventral. RGL Céreste 522.

Fig. 148. Male, terminalia, dorsal. FSL 391921.

Figs. 149-153. Plecia maimensis Gentilini, 1993

Fig. 149. Male, wing. RGL Dauphín 1.

Fig. 150. Male, habitus. RGL Dauphín 122.

Fig. 151. Female, habitus. RGL Bois d'Asson 4.

Fig. 152. Male, terminalia, dorsal. RGL Bois d'Asson 24.

Fig. 153. Female, terminalia, dorsal. RGL Bois d'Asson 4. 
Figs. 154-159. Plecia morio (Heer, 1849)

Fig. 154. Male, wing. MNHN Aix 1398.

Fig. 155. Female, wing. NHMUK In 39860.

Fig. 156. Male, head and thorax, dorsal. MNHN Aix 885.

Fig. 157. Male, head and thorax, ventral. MNHN Aix 1398.

Fig. 158. Female, head. NHMUK In 39831.

Fig. 159. Female, head and thorax. NHMUK In 39860.

Figs. 160-161. Plecia rhenana Heyden \& Heyden, 1865

Fig. 160. Female, wing, NHMUK In 38798.

Fig. 161. Female, head and thorax. NHMUK In 38798.

Figs. 162-164. Bibio aquaesextiae sp.n.

Fig. 162. Male, wing. MNHN Aix 152.

Fig. 163. Male, head and thorax. MNHN Aix 152.

Fig. 164. Male, terminalia. MNHN Aix 152.

Figs. 165-171. Bibio baumbergeri Théobald, 1937

Fig. 165. Male, wing. NMB F2330.

Fig. 166. Female, wing. NMB F1376/1.

Fig. 167. Female, head and thorax. NMB F1377/5.

Fig. 168. Female, fore leg. NMB F1376/1.

Fig. 169. Female, hind leg. NMB F1376/1.

Fig. 170. Female, terminalia, dorsal. NMB F454.

Fig. 171. Male, terminalia. NMB F2183.

Figs. 172-177. Bibio celasensis Théobald, 1937

Fig. 172. Female, wing. MNHN Célas 2.

Fig. 173. Male, habitus. MNHN Monteils 1.

Fig. 174. Female, habitus. MNHN Monteils 101.

Fig. 175. Female, head and thorax. MNHN Monteils 17.

Fig. 176. Male, terminalia, dorsal. MNHN Monteils 1.

Fig. 177. Female, terminalia, lateral. MNHN Monteils 39.

Figs. 178-183. Bibio curtisii Heer, 1856

Fig. 178. Male, wing. ETHZ Aix.

Fig. 179. Female, wing. ETHZ Aix.

Fig. 180. Male, head and thorax. NHMUK In 39833.

Fig. 181. Male, fore leg. NHMUK In 39825.

Fig. 182. Male, hind leg. NHMUK In 39825.

Fig. 183. Male, terminalia, dorsal. MNHN Aix 1062. 
Figs. 184-187. Bibio dislocatus Théobald, 1937

Fig. 184. Female, wing. NMB F1385/3.

Fig. 185. Female, habitus. NMB F1383.

Fig. 186. Female, head and thorax. NMB F1386/2.

Fig. 187. Female, terminalia, ventral. NMB F1385/4.

Figs. 188-197. Bibio major (Oustalet, 1870)

Fig. 188. Male, wing. MNHN Aix Am 67.

Fig. 189. Female, wing. RGL Céreste 655.

Fig. 190. Male, habitus. MNHN Aix 313.

Fig. 191. Male, habitus. RGL Céreste 1029.

Fig. 192. Female, habitus. MNHN Aix 1286.

Fig. 193. Male, head. MNHN Aix 313.

Fig. 194. Male, fore leg. MNHN Aix 313.

Fig. 195. Male, hind leg. MNHN Aix 89.

Fig. 196. Male, terminalia, dorsal. RGL Céreste 34.

Fig. 197. Female, terminalia, ventral. MNHN Aix 2.

Fig. 198-206. Bibio spectabilis Théobald, 1937

Fig. 198. Female, wing. FSL 391911.

Fig. 199. Male, wing. MNHN Aix 1436.

Fig. 200. Male, habitus. MNHN Aix 313.

Fig. 201. Male, head and thorax. MNHN Aix 1436.

Fig. 202. Male, hind leg. MNHN Aix 1377.

Fig. 203. Male, terminalia. MNHN Aix 1436.

Fig. 204. Female, head and thorax. MNHN Aix 1299.

Fig. 205. Female, hind leg. MNHN Aix 845.

Fig. 206. Female, terminalia. MNHN Aix 845.

Figs. 207-211. Bibiodes provincialis sp.n.

Fig. 207. Female, wing. MNHN Aix 23.

Fig. 208. Male, head and thorax. MNHN Aix 1079.

Fig. 209. Female, habitus. MNHN Aix 959.

Fig. 210. Female, hind leg. MNHN Aix 23.

Fig. 211. Male, terminalia. MNHN Aix 1079.

Fig. 212. Bibiodes sp., female, habitus. NMB F2136.

Figs. 213-214. Dilophus luteipennis (Théobald, 1937)

Fig. 213. Female, wing. FSL 391913.

Fig. 214. Female, head and thorax. FSL 391913. 